\title{
Many-to-many space-time network coding for amplify-and-forward cooperative networks: node selection and performance analysis
}

\author{
Mohammed W Baidas ${ }^{1 *}$ and Allen B MacKenzie ${ }^{2}$
}

\begin{abstract}
In this paper, the multinode amplify-and-forward cooperative communications for a network of $N$ nodes is studied via the novel concept of many-to-many space-time network coding (M2M-STNC). Communication under the M2M-STNC scheme is performed over two phases: (1) the broadcasting phase and (2) the cooperation phase. In the former phase, each node broadcasts its data symbol to all the other nodes in the network in its allocated time slot, while in the latter phase, simultaneous transmissions from $N-1$ nodes to a destination node take place in their time slot. In addition, the M2M-STNC scheme with optimal node selection (i.e., M2M-STNC-ONS) is proposed. In this scheme, the optimal relay node is selected based on the maximum harmonic mean value of the source, intermediate, and destination nodes' scaled instantaneous channel gains. Theoretical symbol-error-rate analysis for M-ary phase shift keying (M-PSK) modulation is derived for both the M2M-STNC and M2M-STNC-ONS schemes. Also, the effect of timing synchronization errors and imperfect channel state information on the SER performance and achievable rates is analytically studied. It is shown that the proposed M2M-STNC-ONS scheme outperforms the M2M-STNC scheme and is less sensitive to timing offsets and channel estimation errors. It is envisioned that the M2M-STNC-ONS scheme will serve as a potential many-to-many cooperative communication scheme with applications spanning sensor and mobile ad hoc networks.
\end{abstract}

Keywords: Amplify-and-forward; Channel estimation errors; Network coding; Node selection; Power allocation; Timing synchronization

\section{Introduction}

Network coding has recently emerged as an important design paradigm for wireless networks that allows multinode communications and also improves data distribution and network throughput [1]. Cooperative communications have also attracted much attention in the wireless literature as an effective means of jointly sharing transmissions of distributed single antenna nodes to exploit spatial diversity gains and mitigate channel fading and interference [2]. As most conventional multinode cooperative communication schemes are not directly applicable to information exchange across many geographically distributed nodes, wireless network coding has become increasingly attractive.

\footnotetext{
*Correspondence: baidas@ieee.org

1 Electrical Engineering Department, College of Engineering and Petroleum,

Kuwait University, Safat 13060, Kuwait

Full list of author information is available at the end of the article
}

A few recent works have proposed the use of wireless network coding for multinode cooperative communications in wireless networks. For instance, in [3], the concept of wireless network cocast (WNC) that employs wireless network coding is proposed to achieve aggregate transmission power and delay reduction while achieving incremental diversity in location-aware networks. In [4], complex field network coding (CFNC) was employed to achieve a full diversity gain and a throughput as high as $1 / 2$ symbol per user per channel use. However, research thus far had not fully exploited the joint potential of wireless network coding and cooperative diversity until the introduction of the novel concept of space-time network coding (STNC) [5,6]. In [5], the multipoint-to-point (M2P) and point-to-multipoint (P2M) space-time network codes were proposed to allow multiple source transmissions within a time-division multiple access (TDMA)

\section{黑 Springer}

(C) 2014 Baidas and MacKenzie; licensee Springer. This is an Open Access article distributed under the terms of the Creative Commons Attribution License (http://creativecommons.org/licenses/by/2.0), which permits unrestricted use, distribution, and reproduction in any medium, provided the original work is properly credited. 
framework to a common node and the reverse common node transmission to multiple destinations, respectively. It was also shown that for a network of $N$ nodes deploying M2P-STNC or P2M-STNC, only $2 N$ time slots are required while achieving a diversity order of $N$ per transmitted symbol. In [7], differential space-time network coding (DSTNC) has been proposed for multisource cooperation to counteract the challenges of imperfect synchronization and channel estimation while achieving full diversity. Specifically, the authors analyze the pairwise error probability and derive the design criteria for DSTNC. Anti-eavesdropping space-time network coding (AE-STNC) has been proposed in [8] for secure cooperative communications against eavesdropping, while achieving full diversity. Many-to-one STNC has been proposed in [9] for cluster-based cooperative communications to achieve spatial diversity and improve spectral efficiency. In [10], the symbol error (SER) of STNC is analyzed in independent but not necessarily identically distributed Nakami $-m$ fading channels. Specifically, exact and asymptotic SER expressions are derived for $M$-PSK and $M$-QAM modulations, and the impact of the fading parameter $m$, relay location, power allocation, and non-orthogonal codes on the SER are examined.

In [6], the many-to-many space-time network coding (M2M-STNC) for a network of $N$ decode-and-forward (DF) nodes is proposed to achieve a diversity order of $N-1$ per node over a total of $2 N$ time slots while maintaining a stable network throughput of $1 / 2$ symbol per time slot per node. The operation of the M2M-STNC scheme is based on the assumption of $N-1$ perfectly synchronized simultaneous transmissions in every time slot of the cooperation phase. However, the work in [6] did not analyze the impact of timing offsets on the network performance. In practice, simultaneous transmissions from multiple relay nodes are extremely challenging due to the imperfect timing synchronization. Most research in cooperative communications when focusing on simultaneous transmissions from distributed relay nodes assume perfect timing synchronization $[4,11,12]$. Overlooking the impact of timing synchronization errors could lead to detrimental effects on the network performance [13]. Also, channel state information errors at the receiving nodes are inevitable in practice [14]. Such errors could drastically diminish diversity gains and thus must be carefully characterized.

Based on the foregoing discussion, this work aims at better exploiting the potentials of the M2M-STNC communication scheme for amplify-and-forward (AF) cooperative networks by (1) characterizing the symbol error rate performance for M-ary phase shift keying (M-PSK) modulation and (2) analyzing the impact of timing synchronization errors and channel estimation errors on the SER performance. To reduce the number of simultaneous transmissions while allowing $N$ distributed AF nodes to exchange their data symbols, achieving a diversity order of $N-1$ per node, the M2M-STNC scheme is augmented with optimal node selection (i.e., M2M-STNC-ONS). This work also analyzes the SER performance of the proposed M2M-STNC-ONS scheme and studies the impact of timing synchronization errors and imperfect channel state information.

Although selection in cooperative networks is not a new concept (e.g., see $[15,16])$, the novelty of this work is manifested by augmenting it with a many-to-many communications scheme to achieve full diversity and mitigate the adverse effects of timing offsets and channel estimation errors. The main contributions of this paper are summarized as follows:

- Proposed the M2M-STNC scheme with optimal node selection (i.e., M2M-STNC-ONS) and analytically proved that it achieves full diversity order.

- Analytically studied the effect of timing offsets and channel estimation errors on the performance of the M2M-STNC and M2M-STNC-ONS schemes.

- Demonstrated that the M2M-STNC-ONS scheme is more resistant to timing offsets and channel estimation errors than its counterpart M2M-STNC scheme, in terms of the SER performance as well as achievable rate.

Due to the envisioned merits of the M2M-STNC-ONS scheme, its potential applications may include but are not limited to cluster-based communications for cooperative spectrum sensing and decision fusion in cognitive radio networks [17], and also for reliable and energy-efficient inter- and intra-cluster data gathering in wireless sensor networks [18]. Moreover, the M2M-STNC-ONS scheme can be used for improved network connectivity in clustered mobile ad hoc networks [19]. It is envisioned that the M2M-STNC-ONS scheme will serve as a potential candidate for many-to-many cooperative communications in amplify-and-forward cooperative networks.

In the rest of this paper, the system model of the M2MSTNC scheme is presented in Section 2. The signal model of the proposed M2M-STNC-ONS scheme is discussed in Section 3, while the theoretical symbol error rate of both the M2M-STNC and M2M-STNC-ONS schemes is analyzed in Section 4. The impact of timing offsets and channel estimation errors on the performance of both schemes is characterized in Sections 5, and 6, respectively. Simulation results are contrasted with the analytical results in Section 7. Finally, conclusions are drawn in Section 8.

\section{System model}

The M2M-STNC system model is based on a wireless network with $N$ single antenna amplify-and-forward nodes 
denoted $S_{1}, S_{2}, \ldots, S_{N}$ for $N \geq 4$. Each node $S_{j}$ for $j \in\{1,2, \ldots, N\}$ is assumed to have its own data symbol $x_{j}$ to exchange with all the other $N-1$ nodes in the network. In this work, the channel between any two nodes is modeled as flat Rayleigh fading with additive white Gaussian noise (AWGN). Let $h_{j, i}$ denote a generic channel coefficient representing the channel between any two nodes $S_{j}$ and $S_{i}$ for $j \neq i$, and $h_{j, i}$ is modeled as a zero-mean complex Gaussian random variable with variance $\sigma_{j, i}^{2}$ (i.e., $\left.h_{j, i} \sim \mathcal{C N}\left(0, \sigma_{j, i}^{2}\right)\right)$. The squared channel gain $\left|h_{j, i}\right|^{2}$ is an exponential random variable with mean $\sigma_{j, i}^{2}$. Also, the channel $h_{j, i}$ between nodes $S_{j}$ and $S_{i}$ is assumed to be reciprocal (i.e., $h_{i, j}=h_{j, i}$ ) as in time-division duplexing (TDD) systems, with perfect channel estimation at each node. Moreover, the channel coefficients are assumed to be quasi-static throughout the network operation. Finally, perfect timing synchronization between all the $N$ nodes in the network is assumed.

The cooperative communication between all the nodes (depicted in Figure 1 for $N=4$ ) is performed over a total of $2 N$ time slots and is split into two phases ( $N$ time slots each): (a) the broadcasting phase (BP) and (b) the cooperation phase $(\mathrm{CP})$. The communication under the two phases will be detailed in the following subsections and is expressed in matrix form as

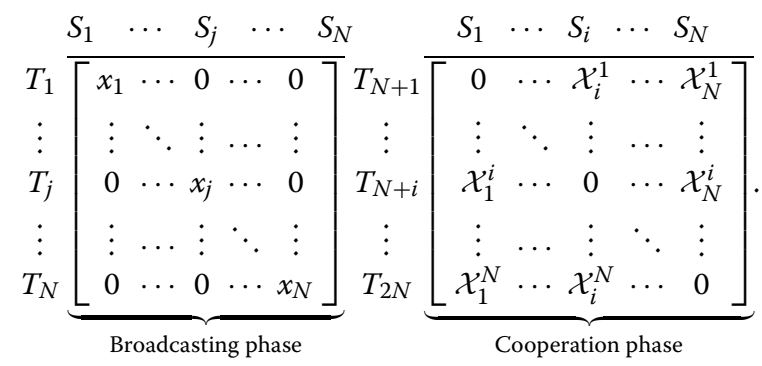

\subsection{Broadcasting phase}

In the broadcasting phase, a source node $S_{j}$ is assigned a time slot $T_{j}$ in which it broadcasts its own data symbol $x_{j}$ to the $N-1$ other nodes $S_{i}$ in the network for $i \in\{1,2, \ldots, N\}$ for $i \neq j$. For source separation at each receiving node, each transmitted symbol $x_{j}$ is spread using a signature waveform $c_{j}(t)$ where it is assumed that each node knows the signature waveforms of all the other nodes. The cross-correlation of $c_{j}(t)$ and $c_{i}(t)$ is $\rho_{j, i}=\left\langle c_{j}(t), c_{i}(t)\right\rangle \triangleq\left(1 / T_{s}\right) \int_{0}^{T_{s}} c_{j}(t) c_{i}^{*}(t) d t$ for $j \neq i$, with $\rho_{j, j}=1$ and $T_{s}$ being the symbol duration. Thus, the signal received at node $S_{i}$ for $i \neq j$ in time slot $T_{j}$ is expressed as

$$
y_{j, i}(t)=\sqrt{P_{j}^{\mathrm{B}}} h_{j, i} x_{j} c_{j}(t)+n_{j, i}(t),
$$

where $P_{j}^{\mathrm{B}}$ is the transmit power in the broadcasting phase at node $S_{j}$, and $h_{j, i}$ is the Rayleigh flat fading channel coefficient between nodes $S_{j}$ and $S_{i}$. Also, $n_{j, i}(t)$ is the additive noise process at node $S_{i}$ due to the signal transmitted by node $S_{j}$, modeled as a zero-mean complex Gaussian random variable with variance $N_{0}$. To extract data symbol $x_{j}$ at node $S_{i}$, the received signal $y_{j, i}(t)$ (given in (2)) is cross-correlated with the signature waveform $c_{j}(t)$ to obtain

$$
y_{j, i}=\left\langle y_{j, i}(t), c_{j}(t)\right\rangle=\sqrt{P_{j}^{\mathrm{B}}} h_{j, i} x_{j}+n_{j, i},
$$

where $n_{j, i} \sim \mathcal{C N}\left(0, N_{0}\right)$. Upon completion of the broadcasting phase, each node $S_{i}$ will have exchanged its data symbol $x_{i}$ with the other nodes and received a set of $N-1$ signals $\left\{y_{j, i}\right\}_{j=1, j \neq i}^{N}$ comprising symbols $x_{1}, \ldots, x_{i-1}, x_{i+1}, \ldots, x_{N}$ for $j \neq i$ from all the other nodes in the network. Node $S_{i}$ then performs a matched filtering operation on each of the received signals $y_{j, i}$, and the signal-to-noise ratio (SNR) at the output of the matched filter is expressed as [2]

$$
\gamma_{j, i}^{\mathrm{BP}}=\frac{P_{j}^{\mathrm{B}}\left|h_{j, i}\right|^{2}}{N_{0}} .
$$

The received signals at each node at the end of the broadcasting phase are expressed as

$$
\mathbf{Y}=\left[\begin{array}{ccccc}
- & y_{2,1} & \cdots & y_{N-1,1} & y_{N, 1} \\
y_{1,2} & - & \cdots & y_{N-1,2} & y_{N, 2} \\
\vdots & \vdots & \ddots & \vdots & \vdots \\
y_{1, N-1} & y_{2, N-1} & \cdots & - & y_{N, N-1} \\
y_{1, N} & y_{2, N} & \cdots & y_{N-1, N} & -
\end{array}\right]
$$

where the $i$ th row represents the signals received at node $S_{i}$, while the $j$ th column represents the signals received in time slot $T_{j}$ from node $S_{j}$.

\subsection{Cooperation phase}

The cooperation phase involves two operations: (1) signal transmission and (2) multinode signal detection, which are discussed in the following subsections, respectively ${ }^{\mathrm{a}}$.

\subsubsection{Signal transmission}

In the cooperation phase, each node $S_{i}$ acts as the destination node in time slot $T_{N+i}$ for $i \in\{1,2, \ldots, N\}$ and receives simultaneous transmissions from the other $N-1$ nodes. In particular, each node $S_{k}$ with $k \neq i$ forms a linearly coded signal $\mathcal{X}_{k}^{i}(t)$ which is composed from the received $N-2$ signals of the $k$ th row of matrix $\boldsymbol{Y}$ in (5), excluding the received signal from node $S_{i}$. Node $S_{k}$ then transmits $\mathcal{X}_{k}^{i}(t)$ which is given by

$$
\mathcal{X}_{k}^{i}(t)=\sum_{\substack{m=1 \\ m \neq i, m \neq k}}^{N} \beta_{m, k, i} y_{m, k} c_{m}(t),
$$




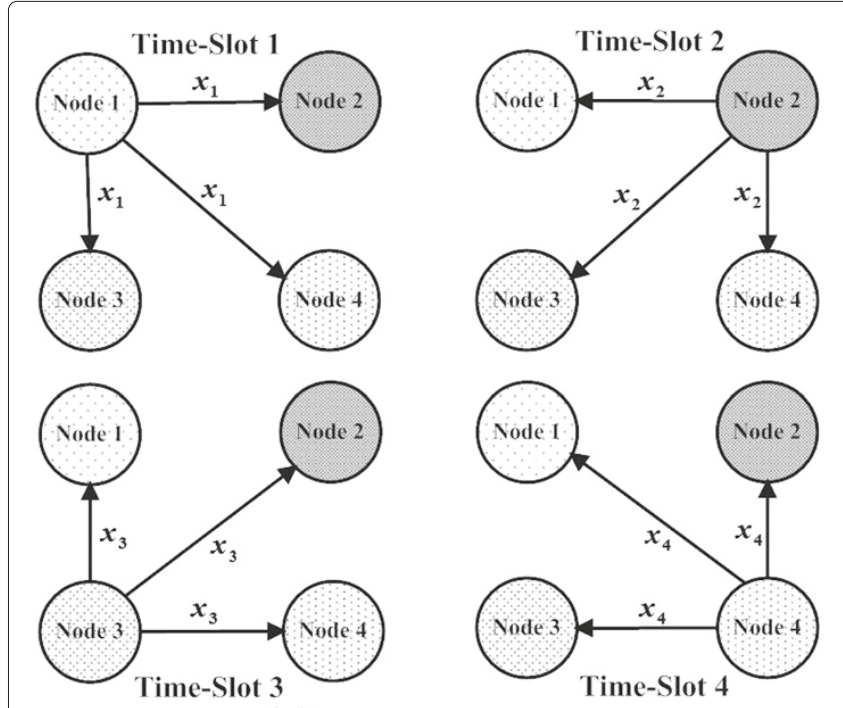

(a) Broadcasting Phase
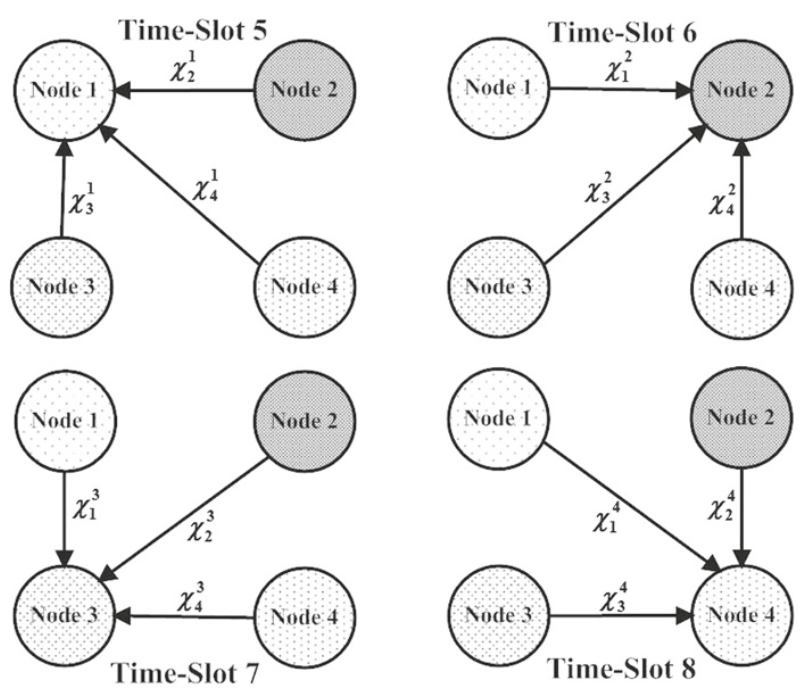

(b) Cooperation Phase

Figure 1 M2M-STNC scheme with $N=4$ nodes. (a) Broadcasting phase. (b) Cooperation phase.

where $c_{m}(t)$ is the signature waveform associated with symbol $x_{m}$, and $\beta_{m, k, i}$ is the normalization factor, as defined by [2]

$$
\beta_{m, k, i}=\sqrt{\frac{P_{m, k, i}^{\mathrm{C}}}{P_{m}^{\mathrm{B}}\left|h_{m, k}\right|^{2}+N_{0}}} .
$$

From (6), it should be noticed that node $S_{k}$ relays the received signals from the other $N-2$ nodes. Moreover, the received signal at node $S_{i}$ during time slot $T_{N+i}$ is given by

$$
\mathcal{Y}_{i}(t)=\sum_{k=1, k \neq i}^{N} h_{k, i} \mathcal{X}_{k}^{i}(t)+w_{i}(t)=\sum_{m=1, m \neq i}^{N} \alpha_{m, i} x_{m} c_{m}(t)+\bar{w}_{i}(t),
$$

where $\alpha_{m, i}$ is defined as

$$
\alpha_{m, i}=\sqrt{P_{m}^{\mathrm{B}}} \sum_{\substack{k=1 \\ k \neq i, k \neq m}}^{N} \beta_{m, k, i} h_{m, k} h_{k, i}
$$

In (8), $w_{i}(t)$ is the zero-mean $N_{0}$ variance additive noise process at node $S_{i}$, and $\bar{w}_{i}(t)$ is the equivalent noise term which can be expressed as

$$
\bar{w}_{i}(t)=w_{i}(t)+\sum_{m=1, m \neq i}^{N} \sum_{\substack{k=1 \\ k \neq i, k \neq m}}^{N} \beta_{m, k, i} h_{k, i} n_{m, k}(t) c_{m}(t) .
$$

The total power at source node $S_{m}$ associated with exchanging symbol $x_{m}$ with the other $N-1$ nodes in the network is given by $P_{m}=P_{m}^{\mathrm{B}}+P_{m}^{\mathrm{C}}$, where $P_{m}^{\mathrm{B}}=\delta_{m}^{\mathrm{B}} P_{m}$ is the broadcast power and $P_{m}^{\mathrm{C}}=\sum_{i=1, i \neq m}^{N} P_{m, i}^{\mathrm{C}}=\delta_{m}^{\mathrm{C}} P_{m}$ is the total cooperative power, with $0<\delta_{m}^{\mathrm{B}} \leq 1$ and $\delta_{m}^{\mathrm{C}}=1-\delta_{m}^{\mathrm{B}}$ being the power allocation fractions to the broadcasting and cooperation phases, respectively. In addition, $P_{m, i}^{\mathrm{C}}$ is the total cooperative power associated with relaying symbol $x_{m}$ to destination node $S_{i}$ for $i \neq m$ such that $P_{m, i}^{\mathrm{C}}=\delta_{m, i}^{\mathrm{C}} P_{m}^{\mathrm{C}}$ with $0 \leq \delta_{m, i}^{\mathrm{C}} \leq 1$. Thus, $P_{m, i}^{\mathrm{C}}$ is given by $P_{m, i}^{\mathrm{C}}=\sum_{\substack{k \neq i, k \neq m \\ k \neq 1}}^{N} P_{m, k, i}^{\mathrm{C}}$ with each relaying node $S_{k}$ for $k \neq m$ and $k \neq i$ being allocated cooperative power $P_{m, k, i}^{\mathrm{C}}=\delta_{m, k, i}^{\mathrm{C}} P_{m, i}^{\mathrm{C}}$ with $0 \leq \delta_{m, k, i}^{\mathrm{C}} \leq 1$. Without any loss of generality, it is assumed that all the transmit power associated with transmitting symbol $x_{m}$ is the same for all the $N$ nodes (i.e., $\left.P_{m}=P=P_{m}^{\mathrm{B}}+P_{m}^{\mathrm{C}}, \forall m \in\{1,2, \ldots, N\}\right)$.

\subsubsection{Multinode signal detection}

Upon receiving signal $\mathcal{Y}_{i}(t)$, a multinode signal detection operation is performed by node $S_{i}$ to extract each of the $N-1$ symbols $x_{j}$, for $j \in\{1,2, \ldots, N\}_{j \neq i}$. This is achieved by passing the received signal $\mathcal{Y}_{i}(t)$ through a matched filter bank (MFB) of $N-1$ branches, matched to the corresponding nodes' signature waveforms $c_{j}(t)$, yielding

$$
\mathcal{Y}_{j, i}=\left\langle\mathcal{Y}_{i}(t), c_{j}(t)\right\rangle=\sum_{m=1, m \neq i}^{N} \alpha_{m, i} x_{m} \rho_{m, j}+\bar{w}_{j, i},
$$

where $\rho_{m, j}$ is the correlation coefficient between $c_{m}(t)$ and $c_{j}(t)$. The output of the MFB can be put in a vector form of all the $N-1 \mathcal{Y}_{i, j}$ 's signals as $\mathcal{Y}_{i}=\boldsymbol{R}_{i} \boldsymbol{A}_{i} \boldsymbol{x}_{i}+$ $\overline{\boldsymbol{w}}_{i}$, where $\mathcal{Y}_{i}=\left[\mathcal{Y}_{1, i}, \ldots, \mathcal{Y}_{i-1, i}, \mathcal{Y}_{i+1, i}, \ldots, \mathcal{Y}_{N, i}\right]^{T}$, and $\boldsymbol{x}_{i}=\left[x_{1}, \ldots, x_{i-1}, x_{i+1}, \ldots, x_{N}\right]^{T}$. In addition, $\overline{\boldsymbol{w}}_{i}=$ $\left[\bar{w}_{1, i}, \ldots, \bar{w}_{i-1, i}, \bar{w}_{i+1, i}, \ldots, \bar{w}_{N, i}\right]^{T} \sim \mathcal{C N}\left(\mathbf{0}, N_{0}\left(\mathbf{I}+\boldsymbol{G}_{i}\right) \boldsymbol{R}_{i}\right)$ 
and $\boldsymbol{R}_{i}, \boldsymbol{A}_{i}$ and $\mathbf{I}, \boldsymbol{G}_{i}$ are $(N-1) \times(N-1)$ matrices with $\mathbf{I}$ being the identity matrix with $\boldsymbol{R}_{i}$ being defined as

$$
\boldsymbol{R}_{i}=\left[\begin{array}{cccccc}
1 & \cdots & \rho_{1,(i-1)} & \rho_{1,(i+1)} & \cdots & \rho_{1, N} \\
\vdots & \ddots & \vdots & \vdots & \cdots & \vdots \\
\rho_{(i-1), 1} & \cdots & 1 & \rho_{(i-1),(i+1)} & \cdots & \rho_{(i-1), N} \\
\rho_{(i+1), 1} & \cdots & \rho_{(i+1),(i-1)} & 1 & \cdots & \rho_{(i+1), N} \\
\vdots & \cdots & \vdots & \vdots & \ddots & \vdots \\
\rho_{N, 1} & \cdots & \rho_{N,(i-1)} & \rho_{N,(i+1)} & \cdots & 1
\end{array}\right],
$$

and the diagonal matrices $A_{i}$ and $G_{i}$ are, respectively, written as

$$
\boldsymbol{A}_{i}=\operatorname{diag}\left[\alpha_{1, i}, \ldots, \alpha_{(i-1), i}, \alpha_{(i+1), i}, \ldots, \alpha_{N, i}\right]
$$

and

$$
\boldsymbol{G}_{i}=\operatorname{diag}\left[g_{1, i}^{2}, \ldots, g_{i-1, i}^{2}, g_{i+1, i}^{2}, \ldots, g_{N, i}^{2}\right]
$$

with $g_{j, i}^{2}$ being defined as $g_{j, i}^{2}=\sum_{\substack{k=1 \\ k \neq i, k \neq j}}^{N} \beta_{j, k, i}^{2}\left|h_{k, i}\right|^{2}$ for $j \neq i$. The received signal vector $\mathcal{Y}_{i}$ can then be decorrelated (assuming matrix $\boldsymbol{R}_{i}$ is invertible) as $\overline{\mathcal{Y}}_{i}=\boldsymbol{R}_{i}^{-1} \mathcal{Y}_{i}=\boldsymbol{A}_{i} \boldsymbol{x}_{i}+$ $\overline{\overline{\boldsymbol{w}}}_{i}$, where $\overline{\overline{\boldsymbol{w}}}_{i}=\boldsymbol{R}_{i}^{-1} \overline{\boldsymbol{w}}_{i}$ and $\overline{\overline{\boldsymbol{w}}}_{i} \sim \mathcal{C N}\left(\mathbf{0}, N_{0} \boldsymbol{R}_{i}^{-1}\left(\mathbf{I}+\boldsymbol{G}_{i}\right)\right)$. Thus, at node $S_{i}$, the decorrelated received signal $\overline{\mathcal{Y}}_{j, i}$ corresponding to symbol $x_{j}$ is obtained as

$$
\overline{\mathcal{Y}}_{j, i}=\sqrt{P_{j}^{\mathrm{B}}}\left(\sum_{\substack{k=1 \\ k \neq i, k \neq j}}^{N} \beta_{j, k, i} h_{j, k} h_{k, i}\right) x_{j}+\overline{\bar{w}}_{j, i},
$$

where $\overline{\bar{w}}_{j, i} \sim \mathcal{C N}\left(0, N_{0} \varrho_{j, i}\left(1+g_{j, i}^{2}\right)\right)$, and $\varrho_{j, i}$ is the $j$ th diagonal element of matrix $\boldsymbol{R}_{i}^{-1}$. Without loss of generality, it is assumed that $\rho_{j, i}=\rho$ for all $j \neq i$ and thus [6]

$$
\varrho_{j, i}=\frac{1+(N-3) \rho}{1+(N-3) \rho-(N-2) \rho^{2}} \triangleq \varrho_{N-1} .
$$

It should be noted that upon the completion of the broadcasting and cooperation phases, each node $S_{i}$ for $i=$ $1,2, \ldots, N$ has received $N-1$ signals containing symbol $x_{j}$ for $j=1,2, \ldots, N$ and $j \neq i$; a direct signal from the source node $S_{j}$ in the broadcasting phase and $N-2$ signals from nodes $S_{m}$ for $m \neq i$ and $m \neq j$, in the cooperation phase. The instantaneous SNR at the output of the matched filter at node $S_{i}$ corresponding to symbol $x_{j}$ is given by

$$
\gamma_{j, i}=\gamma_{j, i}^{\mathrm{BP}}+\gamma_{j, i}^{\mathrm{CP}}
$$

where $\gamma_{j, i}^{\mathrm{BP}}$ is an exponential random variable as in (4) with mean $\lambda_{j, i}^{\mathrm{BP}}=\frac{N_{0}}{P_{j}^{\mathrm{B}} \sigma_{j, i}^{2}}$, and $\gamma_{j, i}^{\mathrm{CP}}$ is

$$
\begin{aligned}
\gamma_{j, i}^{\mathrm{CP}}= & \frac{P_{j}^{\mathrm{B}}}{N_{0} \varrho_{N-1}\left(1+\sum_{\substack{k \neq 1, k \neq j \\
k \neq i}}^{N} \frac{P_{j, k, i}^{\mathrm{C}}\left|h_{k, i}\right|^{2}}{\left.P_{j, k}^{\mathrm{B}}\right|_{j, N_{0}}}\right)} \\
& \times\left(\sum_{\substack{k=1 \\
k \neq i, k \neq j}}^{N} \frac{P_{j, k, i}^{\mathrm{C}}\left|h_{j, k}\right|^{2}\left|h_{k, i}\right|^{2}}{P_{j}^{\mathrm{B}}\left|h_{j, k}\right|^{2}+N_{0}}\right) .
\end{aligned}
$$

It is clear from (18) that $\gamma_{j, i}^{C P}$ is adversely affected by the noise amplification due to the simultaneous transmissions of the $N-2$ nodes. The achievable rate between source node $S_{j}$ and destination node $S_{i}$ is given by

$$
\begin{aligned}
& \mathcal{R}_{j, i}^{\mathrm{M} 2 \mathrm{M}-\mathrm{STNC}}\left(\gamma_{j, i}\right) \\
& =\frac{1}{2 N} \log _{2}\left(1+\frac{P_{j}^{\mathrm{B}}\left|h_{j, i}\right|^{2}}{N_{0}}+\frac{P_{j}^{\mathrm{B}}\left(\sum_{\substack{k=1 \\
k \neq i, k \neq j}}^{N} \frac{P_{j, k i,}^{\mathrm{C}}\left|h_{j, k}\right|^{2}\left|h_{k, i}\right|^{2}}{P_{j}^{\mathrm{B}}\left|h_{j, k}\right|^{2}+N_{0}}\right)}{N_{0} \varrho_{N-1}\left(1+\sum_{\substack{k=1 \\
k \neq i, k \neq j}}^{N} \frac{P_{j, k, i}^{\mathrm{C}}\left|h_{k, i}\right|^{2}}{P_{j}^{\mathrm{B}}\left|h_{j, k}\right|^{2}+N_{0}}\right)}\right),
\end{aligned}
$$

and the total achievable rate by node $S_{j}$ is expressed as $\mathcal{R}_{j}^{\mathrm{M} 2 \mathrm{M}-\mathrm{STNC}}=\sum_{i=1, i \neq j}^{N} \mathcal{R}_{j, i}^{\mathrm{M} 2 \mathrm{M}-\mathrm{STNC}}\left(\gamma_{j, i}\right)$. It should be noted that the M2M-STNC scheme requires stringent timing synchronization between the relaying nodes, and synchronizing all the distributed $N$ nodes, as will be discussed later in this paper, is practically prohibitive.

\section{Space-time network coding with optimal node selection}

When node $S_{i}$ acts as a destination node in its assigned time slot $T_{N+i}$, the intermediate node the transmitted signal of which results in the highest cumulative SNR value for symbol $x_{m}$ of source node $S_{m}$ for $m \neq i$ is selected. Specifically, for each source node, optimal relaying nodes are selected and then all the nodes selected for at least one source node transmit simultaneously. The node selection metric used by the destination node $S_{i}$ to determine the optimal node $S_{k}$ to 'relay' symbol $x_{m}$ received from source node $S_{m}$ for $k \neq i$ and $k \neq m$ is based on the scaled harmonic mean of the instantaneous source, intermediate and destination nodes' scaled channel gains, as follows ${ }^{b}$ $[15,20,21]$

$$
\gamma_{m, k, i} \triangleq \mu_{H}\left(X_{m, k}^{\mathrm{B}} ; X_{m, k, i}^{\mathrm{C}}\right)=\frac{X_{m, k}^{\mathrm{B}} X_{m, k, i}^{\mathrm{C}}}{X_{m, k}^{\mathrm{B}}+X_{m, k, i}^{\mathrm{C}}},
$$

where $X_{m, k}^{\mathrm{B}}=P_{m}^{\mathrm{B}}\left|h_{m, k}\right|^{2}$ and $X_{m, k, i}^{\mathrm{C}}=P_{m, k, i}^{\mathrm{C}}\left|h_{k, i}\right|^{2}$ are exponential random variables corresponding to the 
broadcast transmission of symbol $x_{m}$ from source node $S_{m}$ to intermediate node $S_{k}$ with transmit power $P_{m}^{\mathrm{B}}$ and the cooperative transmission of symbol $x_{m}$ from intermediate node $S_{k}$ to the destination node $S_{i}$ with cooperative transmit power $P_{m, k, i}^{C}=P_{m, i}^{C}$. Thus, the scaled harmonic mean values corresponding to symbol $x_{m}$, for $m \neq i$ at node $S_{k}$ for $k \neq i$ and $k \neq m$ when node $S_{i}$ is the destination node is summarized in a matrix form as

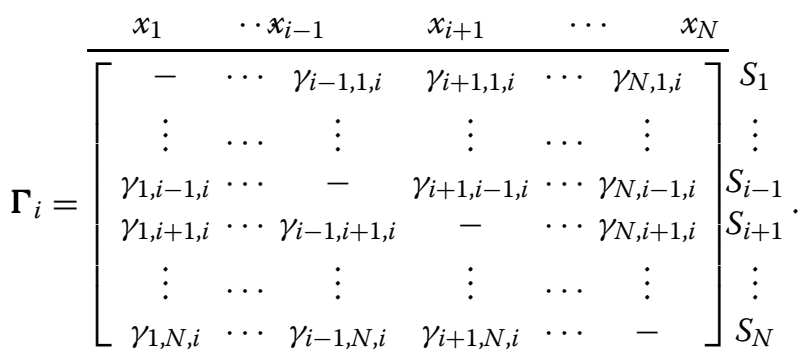

For node $S_{i}$ to receive symbol $x_{m}$ for $m \neq i$, the optimally selected node to forward symbol $x_{m}$ among the $N-2$ nodes that received independent copies of symbol $x_{m}$ during the broadcasting phase is defined by $k_{m, i}^{\mathrm{opt}}=$ $\arg \max _{k=1,2, \ldots, N}\left\{\gamma_{m, k, i}\right\}_{k \neq i, k \neq m}$. Hence, in time slot $T_{N+i}$ for each symbol $x_{m}$ for $m \neq i$, the system reduces to a source node $S_{m}$, a destination node $S_{i}$, and an optimally selected node for the transmission of $x_{m}$. Thus, each symbol $x_{m}$ is associated with a set of indicator functions in the form of $\mathcal{I}_{m, i}=\left\{\mathcal{I}_{m, k, i}\right\}_{k=1, k \neq i, k \neq m}^{N}$, where $\mathcal{I}_{m, k, i}$ for $k \neq i, k \neq m$ acts as a binary indicator function when node $S_{i}$ is the receiving node, while $S_{k}$ is the optimally selected node transmitting signal $y_{m, k}$ corresponding to symbol $x_{m}$. Hence, $\mathcal{I}_{m, k, i}$ is defined by $\mathcal{I}_{m, k, i}=1$ if $k=$ $k_{m, i}^{\mathrm{opt}}$; otherwise, $\mathcal{I}_{m, k, i}=0$.

As before, each node $S_{k}$ then possibly forms a linearly coded signal $\mathcal{Z}_{k}^{i}(t)$ from its received signals in the broadcasting phase and transmits it to node $S_{i}$ during time slot $T_{N+i}$. Specifically, $\mathcal{Z}_{k}^{i}(t)$ is composed from the received signals of the $k$ th row of matrix $Y$ in (5) in the form of

$$
\mathcal{Z}_{k}^{i}(t)=\sum_{\substack{m=1 \\ m \neq i, m \neq k}}^{N} \beta_{m, k, i} y_{m, k} \mathcal{I}_{m, k, i} c_{m}(t)
$$

It should be noted that if node $S_{k}$ is not an optimal node to forward any of the $x_{m}$ for $m \neq i, m \neq k$ data signals to node $S_{i}$, then $\mathcal{Z}_{k}^{i}(t)=0$; otherwise, node $S_{k}$ is an optimal node to forward at least one symbol $x_{m}$ and $\mathcal{Z}_{k}^{i}(t) \neq 0$.
Following the steps of the previous section, the received signal at node $S_{i}$ during time slot $T_{N+i}$ is given by

$$
\hat{\mathcal{Y}}_{i}(t)=\sum_{k=1, k \neq i}^{N} h_{k, i} \mathcal{Z}_{k}^{i}(t)+w_{i}(t)=\sum_{m=1, m \neq i}^{N} \hat{\alpha}_{m, i} x_{m} c_{m}(t) 1+\hat{w}_{i}(t),
$$

where $\hat{\alpha}_{m, i}$ is defined as

$$
\hat{\alpha}_{m, i}=\beta_{m, \mathrm{opt}, i} \bar{h}_{m, \mathrm{opt}, i} \sqrt{P_{m}^{\mathrm{B}}} \hat{h}_{m, \mathrm{opt}, i},
$$

with $\bar{h}_{m, \text { opt }, i}$ being the channel coefficient between the source node $S_{m}$ and the optimally selected node to forward symbol $x_{m}$ to node $S_{i}$ for $m \neq i$, as implied by $k=k_{m, i}^{\mathrm{opt}}$, and $\beta_{m, \mathrm{opt}, i}$ is the scaling factor defined in (7). Also, $\hat{h}_{m, \text { opt }, i}$ is the channel coefficient between the optimally selected node and node $S_{i}$ for the transmission of symbol $x_{m}$ for $m \neq i$. In (23), $\hat{w}_{i}(t)$ is the equivalent noise term which can be expressed as

$$
\hat{w}_{i}(t)=w_{i}(t)+\sum_{m=1, m \neq i}^{N} \beta_{m, \mathrm{opt}, i} \hat{h}_{m, \mathrm{opt}, i} n_{m, \mathrm{opt}, i}(t) c_{m}(t),
$$

where $n_{m, o p t, i}$ is the noise sample at the optimally selected node by node $S_{i}$ for the transmission of symbol $x_{m}$, for $m \neq i$. It should be noted that under the M2M-STNCONS scheme, the total cooperative transmit power associated with relaying symbol $x_{m}$ to node $S_{i}$ is set to $P_{m, \mathrm{opt}, i}^{\mathrm{C}}=$ $P_{m, i}^{\mathrm{C}}=\delta_{m, i}^{\mathrm{C}} P_{m}^{\mathrm{C}}$, where $P_{m, \mathrm{opt}, i}^{\mathrm{C}}$ is the cooperative transmit power allocated to the optimally selected node. Thus, the total power associated with transmitting symbol $x_{m}$ is given by $P=P_{m}^{\mathrm{B}}+\sum_{i=1, i \neq m}^{N} P_{m, \mathrm{opt}, i}^{\mathrm{C}}$.

To extract symbol $x_{j}$, the received signal $\hat{\mathcal{Y}}_{i}(t)$ is passed through a MFB, and the output of the $j$ th branch is expressed as $\hat{\mathcal{Y}}_{j, i}=\sum_{m=1, m \neq i}^{N} \hat{\alpha}_{m, i} x_{m} \rho_{m, j}+\hat{w}_{j, i}$ which in vector form is expressed as $\hat{\mathcal{Y}}_{i}=\boldsymbol{R}_{i} \hat{\boldsymbol{A}}_{i} \boldsymbol{x}_{i}+\hat{\boldsymbol{w}}_{i}$. In particular, $\hat{\boldsymbol{w}}_{i}=\left[\hat{w}_{1, i}, \ldots, \hat{w}_{i-1, i}, \hat{w}_{i+1, i}, \ldots, \hat{w}_{N, i}\right]^{T} \sim \mathcal{C N}\left(\mathbf{0}, N_{0}(\mathbf{I}+\right.$ $\left.\hat{\boldsymbol{G}}_{i}\right) \boldsymbol{R}_{i}$ ), with $\boldsymbol{R}_{i}$ being defined in (12), while $\hat{\boldsymbol{A}}_{i}$ and $\hat{\boldsymbol{G}}_{i}$ are defined as

$$
\hat{A}_{i}=\operatorname{diag}\left[\hat{\alpha}_{1, i}, \ldots, \hat{\alpha}_{(i-1), i}, \hat{\alpha}_{(i+1), i}, \ldots, \hat{\alpha}_{N, i}\right],
$$

and

$$
\hat{\mathbf{G}}_{i}=\operatorname{diag}\left[\hat{g}_{1, i}^{2}, \ldots, \hat{g}_{i-1, i}^{2}, \hat{g}_{i+1, i}^{2}, \ldots, \hat{g}_{N, i}^{2}\right],
$$

with $\hat{g}_{j, i}^{2}$ being defined as $\hat{g}_{j, i}^{2}=\beta_{j, \mathrm{opt}, i}^{2}\left|\hat{h}_{j, \mathrm{opt}, i}\right|^{2}$ for $j \neq i$. The decorrelated signal $\hat{\mathcal{Y}}_{j, i}$ is given by

$$
\hat{\mathcal{Y}}_{j, i}=\beta_{j, \mathrm{opt}, i} \bar{h}_{j, \mathrm{opt}, i} \sqrt{P_{j}^{\mathrm{B}}} \hat{h}_{j, \mathrm{opt}, i} x_{j}+\hat{\hat{w}}_{j, i}
$$

where $\hat{\hat{w}}_{j, i} \sim \mathcal{C N}\left(0, N_{0} \varrho_{N-1}\left(1+\beta_{j, \text { opt }, i}^{2}\left|\hat{h}_{j, \text { opt }, i}\right|^{2}\right)\right)$. At the end of the broadcasting and cooperation phases, two signals comprising symbol $x_{j}$ are received at node $S_{i}$; the 
first comes from the direct transmission in the broadcasting phase, while the other is from the optimally selected node in the cooperation phase. At the output of the matched filter, the instantaneous SNR is given by

$$
\hat{\gamma}_{j, i}=\gamma_{j, i}^{\mathrm{BP}}+\hat{\gamma}_{j, i}^{\mathrm{CP}}
$$

where $\hat{\gamma}_{j, i}^{\mathrm{CP}}$ is given by

$$
\hat{\gamma}_{j, i}^{\mathrm{CP}}=\frac{P_{j, i}^{\mathrm{C}} P_{j}^{\mathrm{B}}\left|\hat{h}_{j, \mathrm{opt}, i}\right|^{2}\left|\bar{h}_{j, \mathrm{opt}, i}\right|^{2}}{N_{0} \varrho_{N-1}\left(P_{j}^{\mathrm{B}}\left|\bar{h}_{j, \mathrm{opt}, i}\right|^{2}+P_{j, i}^{\mathrm{C}}\left|\hat{h}_{j, \mathrm{opt}, i}\right|^{2}+N_{0}\right)},
$$

which at high SNR can be tightly approximated as [2]

$$
\begin{aligned}
\overline{\hat{\gamma}}_{j, i}^{\mathrm{CP}} & \simeq \frac{P_{j, i}^{\mathrm{C}} P_{j}^{\mathrm{B}}\left|\hat{h}_{j, \mathrm{opt}, i}\right|^{2}\left|\bar{h}_{j, \mathrm{opt}, i}\right|^{2}}{N_{0} \varrho_{N-1}\left(P_{j}^{\mathrm{B}}\left|\bar{h}_{j, \mathrm{opt}, i}\right|^{2}+P_{j, i}^{\mathrm{C}}\left|\hat{h}_{j, \mathrm{opt}, i}\right|^{2}\right)} \\
& =\mu_{H}\left(X_{j, \mathrm{opt}}^{\mathrm{B}} ; X_{j, \mathrm{opt}, i}^{\mathrm{C}}\right),
\end{aligned}
$$

where it can be verified that $\overline{\hat{\gamma}}_{j, i}^{\mathrm{CP}}$ is the scaled harmonic mean of two exponential random variables

$$
X_{j, \mathrm{opt}}^{\mathrm{B}}=\frac{P_{j}^{\mathrm{B}}\left|\bar{h}_{j, \mathrm{opt}, i}\right|^{2}}{N_{0} \varrho_{N-1}} \text { and } X_{j, \mathrm{opt}, i}^{\mathrm{C}}=\frac{P_{j, i}^{\mathrm{C}}\left|\hat{h}_{j, \mathrm{opt}, i}\right|^{2}}{N_{0} \varrho_{N-1}},
$$

with means $\lambda_{j, \mathrm{opt}, i}^{\mathrm{B}}=\frac{N_{0} \varrho_{N-1}}{P_{j}^{\mathrm{B}} \bar{\sigma}_{j, \mathrm{opt}, i}^{2}}$ and $\lambda_{j, \mathrm{opt}, i}^{\mathrm{C}}=\frac{N_{0} \varrho_{N-1}}{P_{j, i}^{\mathrm{C}} \hat{\sigma}_{j, \mathrm{opt}, i}^{2}}$, respectively. Note that $\overline{\hat{\gamma}}_{j, i}^{\mathrm{CP}}$ corresponds to the optimally selected node with the maximum harmonic mean. The means of $\gamma_{j, i}^{\mathrm{BP}}, X_{j, \mathrm{opt}}^{\mathrm{B}}$ and $X_{j, \mathrm{opt}, i}^{\mathrm{C}}$ are redefined, respectively, as $\lambda_{j, i}^{\mathrm{BP}}=$ $\frac{N_{0}}{\delta_{j}^{\mathrm{B}} P \sigma_{j, i}^{2}}, \lambda_{j, \mathrm{opt}, i}^{\mathrm{B}}=\frac{N_{0} \varrho_{N-1}}{\delta_{j}^{\mathrm{B} P} \bar{\sigma}_{\bar{j}, \mathrm{opt}, i}}$ and $\lambda_{j, \mathrm{opt}, i}^{\mathrm{C}}=\frac{N_{0} \varrho_{-1}}{\delta_{j, i}^{\mathrm{C}}\left(1-\delta_{j}^{\mathrm{B}}\right) P \hat{\sigma}_{j, \mathrm{opt}, i}^{2}}$, $\forall i, j \in\{1,2, \ldots, N\}_{j \neq i}$. Thus, $\hat{\gamma}_{j, i}$ is redefined as $\overline{\hat{\gamma}}_{j, i}=$ $\gamma_{j, i}^{\mathrm{BC}}+\overline{\hat{\gamma}}_{j, i}^{\mathrm{CP}}$. The achievable rate between source node $S_{j}$ and destination node $S_{i}$ under the M2M-STNC-ONS scheme is given by

$$
\begin{aligned}
& \mathcal{R}_{j, i}^{\mathrm{M} 2 \mathrm{M}-S T N C-O N S}\left(\hat{\gamma}_{j, i}\right) \\
& =\frac{1}{2 N} \log _{2}\left(1+\frac{P_{j}^{\mathrm{B}}\left|h_{j, i}\right|^{2}}{N_{0}}+\frac{P_{j, i}^{\mathrm{C}} P_{j}^{\mathrm{B}}\left|\hat{h}_{j, \mathrm{op}, i}\right|^{2}\left|\bar{h}_{j, \mathrm{opt}, i}\right|^{2}}{N_{0} \varrho_{N-1}\left(P_{j}^{\mathrm{B}}\left|\bar{h}_{j, \mathrm{op}, i}\right|^{2}+P_{j, i}^{\mathrm{C}}\left|\hat{h}_{j, \mathrm{opt}, i}\right|^{2}+N_{0}\right)}\right) .
\end{aligned}
$$

Thus $\mathcal{R}_{j}^{\mathrm{M} 2 \mathrm{M}-\mathrm{STNC}-\mathrm{ONS}}=\sum_{i=1, i \neq j}^{N} \mathcal{R}_{j, i}^{\mathrm{M} 2 \mathrm{M}-\mathrm{STNC}-\mathrm{ONS}}\left(\hat{\gamma}_{j, i}\right)$ is the total rate achievable by node $S_{j}$.

\section{Symbol error rate performance analysis}

\subsection{M2M-STNC}

In general, the SER for M-PSK modulation conditional on the channel state information (CSI) for SNR $\gamma$ is given by [22]

$$
\Psi_{\mid\{h\}}(\gamma)=\frac{1}{\pi} \int_{0}^{(M-1) \pi / M} \exp \left(-\frac{b_{\mathrm{psk}} \gamma}{\sin ^{2} \theta}\right) d \theta,
$$

where $b_{\mathrm{psk}}=\sin ^{2}(\pi / M)$. The derived instantaneous SNR due to the cooperative transmission $\gamma_{j, i}^{\mathrm{CP}}$ in (18) is extremely difficult to manipulate [5]. Thus, only the conditional SER of symbol $x_{j}$ detected at node $S_{i}($ for $i \neq j)$ is provided, which can be evaluated numerically as

$$
P_{\mathrm{SER}}^{\mathrm{M} 2 \mathrm{M}-\mathrm{STNC}}=\Psi_{\mid\left\{h_{j, i}\right\}_{j, i=1, j \neq i}^{N}}\left(\gamma_{j, i}^{\mathrm{BP}}+\gamma_{j, i}^{\mathrm{CP}}\right),
$$

where $\gamma_{j, i}^{\mathrm{BP}}+\gamma_{j, i}^{\mathrm{CP}}=\gamma_{j, i}$, as defined in (17).

\subsection{M2M-STNC-ONS}

Denoting the moment generating function (MGF) of a random variable $Z$ with probability density function (PDF) $p_{Z}(z)$ as

$$
\mathcal{M}_{Z}(s)=\int_{-\infty}^{\infty} e^{-s z} p_{Z}(z) d z,
$$

and averaging the conditional SER over the Rayleigh fading channel statistics, the approximate SER expression is given by

$$
\begin{aligned}
\tilde{P}_{\mathrm{SER}}^{\mathrm{M} 2 \mathrm{M}-\mathrm{STNC}-\mathrm{ONS}}= & \Psi\left(\gamma_{j, i}^{\mathrm{BP}}+\overline{\hat{\gamma}}_{j, i}^{\mathrm{C}}\right)=\frac{1}{\pi} \int_{0}^{(M-1) \pi / M} \\
& \times \mathcal{M}_{\gamma_{j, i}^{\mathrm{BP}}}\left(\frac{b_{\mathrm{psk}}}{\sin ^{2} \theta}\right) \mathcal{M}_{\overline{\hat{\gamma}}_{j, i}^{\mathrm{CP}}}\left(\frac{b_{\mathrm{psk}}}{\sin ^{2} \theta}\right) d \theta,
\end{aligned}
$$

where the approximation is due to the use of $\overline{\hat{\gamma}}_{j, i}^{\mathrm{CP}}$ in (31) instead of $\hat{\gamma}_{j, i}^{\mathrm{CP}}$ in (30). Additionally, $\mathcal{M}_{\gamma_{j, i}^{\mathrm{BP}}}(s)$ is defined as [2]

$$
\mathcal{M}_{\gamma_{j, i}^{\mathrm{BP}}}(s)=\frac{1}{1+s \delta_{j}^{\mathrm{B}} P \sigma_{j, i}^{2} / N_{0}} .
$$

To determine the MGF of $\overline{\hat{\gamma}}_{j, i}^{\mathrm{CP}}$, the cumulative distribution function (CDF) of $\overline{\hat{\gamma}}_{j, i}^{\mathrm{CP}}$ is derived as

$$
P_{\hat{\gamma}_{j, i}^{\mathrm{CP}}}(\gamma)=\operatorname{Pr}\left[\max _{\substack{k=1,2, \ldots, N \\ k \neq i, k \neq j}} \hat{\gamma}_{j, k, i} \leq \gamma\right]=\prod_{\substack{k=1 \\ k \neq i, k \neq j}}^{N} P_{\hat{\gamma}_{j, k, i}}(\gamma),
$$

where

$$
\hat{\gamma}_{j, k, i}=\frac{P_{j, i}^{\mathrm{C}} P_{j}^{\mathrm{B}}\left|h_{j, k}\right|^{2}\left|h_{k, i}\right|^{2}}{N_{0} \varrho_{N-1}\left(P_{j}^{\mathrm{B}}\left|h_{j, k}\right|^{2}+P_{j, i}^{\mathrm{C}}\left|h_{k, i}\right|^{2}\right)},
$$

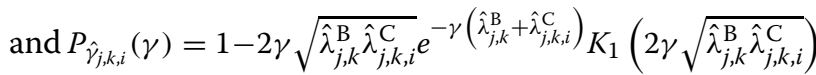
with $\hat{\lambda}_{j, k}^{\mathrm{B}}$ and $\hat{\lambda}_{j, k, i}^{\mathrm{C}}$ being defined as $\hat{\lambda}_{j, k}^{\mathrm{B}}=\frac{N_{0} \varrho_{N-1}}{\delta_{j}^{\mathrm{B} P \sigma_{j, k}^{2}}}$ and $\hat{\lambda}_{j, k, i}^{\mathrm{C}}=\frac{N_{0} \varrho_{N-1}}{\delta_{j, i}^{\mathrm{C}}\left(1-\delta_{j}^{\mathrm{B}}\right) P \sigma_{k, i}^{2}}$, respectively. Also, $K_{1}(\cdot)$ is the firstorder modified Bessel function of the second kind [23]. At high SNR, $K_{1}(\cdot)$ can be approximated for small $x$ as $K_{1}(x) \approx 1 / x$ [23], and thus the CDF of $\hat{\gamma}_{j, k, i}$ simplifies 
to $P_{\hat{\gamma}_{j, k, i}}(\gamma) \approx 1-e^{-\gamma\left(\hat{\lambda}_{j, k}^{\mathrm{B}}+\hat{\lambda}_{j, k, i}^{\mathrm{C}}\right)}$. For convenience, define $\hat{\lambda}_{j, k, i}^{\mathrm{BC}} \triangleq \hat{\lambda}_{j, k}^{\mathrm{B}}+\hat{\lambda}_{j, k, i}^{\mathrm{C}}=\frac{N_{0} \varrho_{N-1}}{P} \Phi_{j, k, i}$, where

$$
\Phi_{j, k, i}=\frac{\delta_{j}^{\mathrm{B}} \sigma_{j, k}^{2}+\delta_{j, i}^{\mathrm{C}}\left(1-\delta_{j}^{\mathrm{B}}\right) \sigma_{k, i}^{2}}{\delta_{j, i}^{\mathrm{C}}\left(1-\delta_{j}^{\mathrm{B}}\right) \delta_{j}^{\mathrm{B}} \sigma_{j, k}^{2} \sigma_{k, i}^{2}} .
$$

Therefore, the PDF of $\overline{\hat{\gamma}}_{j, i}^{\mathrm{CP}}$ can be obtained as

$$
p_{\bar{\gamma}_{j, i}^{\mathrm{CP}}}(\gamma)=\sum_{\substack{m=1 \\ m \neq i, m \neq j}}^{N} p_{\hat{\gamma}_{j, m, i}}(\gamma) \prod_{\substack{k=1, k \neq m \\ k \neq i, k \neq j}}^{N}\left(1-e^{-\gamma \hat{\lambda}_{j, k, i}^{\mathrm{BC}}}\right),
$$

where $p_{\hat{\gamma}_{j, m, i}}(\gamma)=\hat{\lambda}_{j, m, i}^{\mathrm{BC}} e^{-\gamma \hat{\lambda}_{j, m, i}^{\mathrm{BC}}}$ is the PDF of $\hat{\gamma}_{j, m, i}$. Using (42) to determine the MGF of $\overline{\hat{\gamma}}_{j, i}^{\mathrm{CP}}$ is quite difficult [20]; however, a useful relationship between the CDF of a random variable $X$ and its MGF exists and is given by $\mathcal{M}_{X}(s)=s \mathcal{L}\left\{P_{X}(x)\right\}$, with $\mathcal{L}\{\cdot\}$ being the Laplace transform of the parameter CDF [23]. Hence, by substituting $P_{\hat{\gamma}_{j, k, i}}(\gamma) \approx 1-e^{-\gamma\left(\hat{\lambda}_{j, k}^{\mathrm{B}}+\hat{\lambda}_{j, k, i}^{\mathrm{C}}\right)}$ into (39), expanding the resulting product and then taking the Laplace transform, the MGF of $\overline{\hat{\gamma}}_{j, i}^{C P}$ can be shown to be

$$
\begin{aligned}
\mathcal{M}_{\overline{\hat{\gamma}}_{j, i}^{\mathrm{CP}}}(s)= & -\sum_{\substack{k=1 \\
k \neq i, k \neq j}}^{N} \frac{s}{s+\hat{\lambda}_{j, k, i}^{\mathrm{BC}}}+\sum_{\substack{k=1 \\
k \neq i, k \neq j}}^{N} \sum_{\substack{m=1, m \neq k \\
m \neq i, m \neq j}}^{N}{ } \times \frac{s}{s+\hat{\lambda}_{j, k, i}^{\mathrm{BC}}+\hat{\lambda}_{j, m, i}^{\mathrm{BC}}}-\cdots+\frac{(-1)^{N} s}{s+\sum_{\substack{k=1 \\
k \neq i, k \neq j}}^{N} \hat{\lambda}_{j, k, i}^{\mathrm{BC}}} .
\end{aligned}
$$

Thus, by substituting (38) and (43) into (37), the approximate SER performance for symbol $x_{j}$ detected at node $S_{i}$ for $i \neq j$ can be determined using

$$
\begin{aligned}
\tilde{P}_{\mathrm{SER}}^{\mathrm{M} 2 \mathrm{M}-\mathrm{STNC}-\mathrm{ONS}} \approx & \frac{1}{\pi} \int_{0}^{(M-1) \pi / M} \frac{1}{\left(1+\frac{b_{\mathrm{psk}} \delta_{j}^{\mathrm{B}} P \sigma_{j, i}^{2}}{\sin ^{2}(\theta) N_{0}}\right)} \\
& \times\left(1-\sum_{\substack{k=1 \\
k \neq i, k \neq j}}^{N} \frac{\frac{P b_{\mathrm{PSK}}}{\sin ^{2}(\theta) N_{0} \varrho_{N-1}}}{\frac{P b_{\mathrm{PSK}}}{\sin ^{2}(\theta) N_{0} \varrho_{N-1}}+\Phi_{j, k, i}}+\cdots\right. \\
& \left.+\frac{(-1)^{N} \frac{P b_{\mathrm{PSK}}}{\sin ^{2}(\theta) N_{0} \varrho_{N-1}}}{\frac{P b_{\mathrm{PSK}}}{\sin ^{2}(\theta) N_{0} \varrho_{N-1}}+\sum_{\substack{k \neq 1 \\
k \neq i, k \neq j}}^{N} \Phi_{j, k, i}}\right) d \theta .
\end{aligned}
$$

\subsubsection{Asymptotic upper bound}

An asymptotic upper bound on the SER performance is derived by first noticing that at high SNR, the MGF of $\gamma_{j, i}^{\mathrm{BP}}$ given in (38) can be asymptotically upper bounded as [2]

$$
\mathcal{M}_{\gamma_{j, i}^{\mathrm{BP}}}(s) \lesssim \frac{N_{0}}{s \delta_{j}^{\mathrm{B}} P \sigma_{j, i}^{2}}
$$

Second, an asymptotic upper bound for $\mathcal{M}_{\bar{\gamma}_{j, i}^{\mathrm{CP}}}(s)$ at high SNR can be determined by approximating $e^{x} \simeq(1+x)$ when $x \rightarrow 0$ in the PDF of $\overline{\hat{\gamma}}_{j, i}^{\mathrm{CP}}$ defined in (31), which is now given by

$$
p_{\overline{\hat{\gamma}}_{j, i}^{\mathrm{CP}}}(\gamma) \approx \sum_{\substack{m=1 \\ m \neq i, m \neq j}}^{N} \lambda_{j, m, i}^{\mathrm{BC}}\left(1-\gamma \lambda_{j, m, i}^{\mathrm{BC}}\right) \gamma^{N-3} \prod_{\substack{k=1, k \neq m \\ k \neq i, k \neq j}}^{N} \lambda_{j, k, i}^{\mathrm{BC}} .
$$

Since $\lambda_{j, k, i}^{\mathrm{BC}}=\frac{N_{0} \varrho_{N-1}}{P} \Phi_{j, k, i}$, so by substituting (46) into (36), it can be shown that

$$
\mathcal{M}_{\overline{\hat{\gamma}}_{j, i}^{\mathrm{CP}}}(s) \lesssim(N-2) !\left(\frac{N_{0} r_{N-1}}{s P}\right)^{N-2} \prod_{\substack{k=1 \\ k \neq i, k \neq j}}^{N} \Phi_{j, k, i} .
$$

Finally, by substituting (45) and (47) into (37), the asymptotic upper-bound SER expression is obtained as

$$
\begin{aligned}
\bar{P}_{\mathrm{UB}-\mathrm{SER}}^{\mathrm{M} 2 \mathrm{M}-\mathrm{STN}-\mathrm{ONS}} \lesssim & \left(\frac{N_{0}}{P}\right)^{N-1} \frac{(N-2) ! \Theta(N-1)\left(\varrho_{N-1}\right)^{N-2}}{\delta_{j}^{\mathrm{B}} \sigma_{j, i}^{2} b_{\mathrm{PSK}}^{N-1}} \\
& \times \prod_{\substack{k=1 \\
k \neq i, k \neq j}}^{N} \Phi_{j, k, i},
\end{aligned}
$$

with $\Theta(N-1)$ being defined as $\Theta(N-1)=\frac{1}{\pi} \int_{0}^{(M-1) \pi / M}$ $\left(\sin ^{2}(\theta)\right)^{N-1} d \theta$.

\subsubsection{Diversity order analysis}

The diversity order is given by $\Gamma=-\lim _{\mathrm{SNR} \rightarrow \infty}$ $\log \left(\bar{P}_{\mathrm{UB}-\mathrm{MER}}^{\mathrm{M} 2 \mathrm{M} \text {-STC-ONS }}\right) / \log (\mathrm{SNR})$, where SNR $=P / N_{0}$ [2]. Clearly, the M2M-STNC-ONS scheme achieves a full diversity order of $\Gamma=N-1$ per node.

It is noteworthy that the concept of many-to-many space-time network coding with optimal node selection allows us to achieve full diversity of $N-1$ per network node with only $2 N$ time slots. In conventional TDMAbased cooperative communications (i.e., without network coding and multiple-access transmissions), a total of $N^{2}$ time slots is required to achieve full diversity. Clearly, our scheme is more bandwidth efficient than conventional cooperative communication systems.

\section{Timing synchronization analysis}

It is well known that due to the diagonal structure of the broadcasting phase, as shown in (1), the problem of perfect timing synchronization is alleviated since within the TDMA framework, only one source node is allowed to transmit at any one time [24]. Moreover, the analysis so far assumed perfect 'in-phase' synchronization among the transmitting nodes in the cooperation phase. However, 
simultaneous transmissions of the different nodes during the cooperation phase impose a major practical challenge, especially for a large number of the transmitting nodes distributed over a large network area. Clock mismatches of the geographically distributed nodes result in different transmission times. Also, the lack of tracking at the receiving node for all the other cooperative nodes and the lack of compensation for propagation delays can have detrimental effects on the network performance. Thus, this section aims at analyzing the degradation in the SER performance of the M2M-STNC and M2M-STNC-ONS schemes due to the timing offsets between the nodes in the cooperation phase.

\subsection{Signal model under M2M-STNC scheme}

In the cooperation phase, consider the scenario where node $S_{i}$ is the receiving node while the remaining distributed nodes $S_{m}$ for $m \in\{1,2, \ldots, N\}_{m \neq i}$ transmit asynchronously. Let $\tau_{i, m}$ be the time offset for each transmitting node $S_{m}$ during the $i$ th time slot. Also, assume that each distributed node initiates and terminates its transmissions within $T_{s}$ time units of each other within each TDMA time slot. Moreover, the effect of the different propagation delays is manifested in the form of superposition of pulses from each node $S_{m}$ for $m \in\{1,2, \ldots, N\}_{m \neq i}$ that are shifted by $\tau_{i, m}$. This implies that neighboring symbols will introduce intersymbol interference (ISI) to the desired symbol. In this work, only the ISI contribution from the neighboring symbols to the desired symbol is considered, while higher-order terms are neglected due to their smaller effect [14]. From (8), the received signal at node $S_{i}$ during the $i$ th time slot is expressed as ${ }^{\mathrm{c}}[25,26]$

$$
\mathcal{Y}_{i}(t)=\sum_{m=1, m \neq i}^{N} \alpha_{m, i} \sum_{l=-1}^{1} x_{m}(n) c_{m}\left(t-l T_{s}-\tau_{i, m}\right)+\bar{w}_{i}(t),
$$

where $\alpha_{m, i}$ is defined in (9), and $\bar{w}_{i}(t)$ is written as

$$
\begin{aligned}
\bar{w}_{i}(t)= & w_{i}(t)+\sum_{m=1, m \neq i}^{N} \sum_{\substack{k=1 \\
k \neq i, k \neq m}}^{N} \beta_{m, k, i} h_{k, i} \\
& \times \sum_{l=-1}^{1} n_{m, k}\left(t-l T_{s}\right) c_{m}\left(t-l T_{s}-\tau_{i, m}\right) .
\end{aligned}
$$

Without loss of generality, the random time shifts between the $N-1$ nodes and the receiving node $S_{i}$ are ordered such that $0 \leq \tau_{i, 1} \leq \ldots \leq \tau_{i, i-1} \leq \tau_{i, i+1} \leq \ldots \leq \tau_{i, N}<T_{s}$. As before, the received signal is then fed into a bank of $(N-1)$ filters, matched to the nodes' signature waveforms, and sampled at $t=l T_{s}+\Delta_{i}$, where $\Delta_{i}$ is the timing shift chosen by the receiving node $S_{i}$ to compensate for the average delay of the transmitting nodes. Thus, the received signal is given by [27]

$\mathcal{Y}_{j, i}(n)=\left\langle\mathcal{Y}_{i}(t), c_{j}(t)\right\rangle=\int_{l T_{s}+\Delta_{i}}^{l T_{s}+T_{s}+\Delta_{i}} \mathcal{Y}_{i}(t) c_{j}^{*}\left(t-l T_{s}-\Delta_{i}\right) d t$,

with $c_{j}(t)$ being zero outside the duration of $T_{s}$ time units. Define the $(N-1) \times(N-1)$ cross-correlation matrix $\boldsymbol{R}_{i}(l)$ whose entries are modeled for $l=1, l=0$, and $l=-1$ as [28]

$$
\begin{aligned}
& \tilde{\rho}_{m, j}^{(1)} \\
& =\left\{\begin{array}{ll}
\frac{1}{T_{s}} \int_{\Delta_{i}}^{\tau_{i, m}} c_{m}\left(t-\tau_{i, m}\right) c_{j}^{*}\left(t-\Delta_{i}\right) d t=\rho_{m, j}\left(\frac{\tau_{i, m}-\Delta_{i}}{T_{s}}\right), & \tau_{i, m}>\Delta_{i} \\
0, & \text { otherwise }
\end{array},\right.
\end{aligned}
$$

$$
\begin{aligned}
\bar{\rho}_{m, j}^{(0)} & =\frac{1}{T_{s}} \int_{\max \left(\tau_{i, m}, \Delta_{i}\right)}^{\min \left(\tau_{i, m}, \Delta_{i}\right)+T_{s}} c_{m}\left(t-\tau_{i, m}\right) c_{j}^{*}\left(t-\Delta_{i}\right) d t \\
& =\left\{\begin{array}{l}
\rho_{m, j}\left(1-\frac{\Delta_{i}-\tau_{i, m}}{T_{s}}\right), \Delta_{i} \geq \tau_{i, m} \\
\rho_{m, j}\left(1-\frac{\tau_{i, m}-\Delta_{i}}{T_{s}}\right), \Delta_{i}<\tau_{i, m}
\end{array}\right.
\end{aligned}
$$

and

$$
\begin{aligned}
& \tilde{\rho}_{m, j}^{(-1)} \\
& =\left\{\begin{array}{ll}
\frac{1}{T_{s}} \int_{\tau_{i, m}}^{\Delta_{i}} c_{m}\left(t-\tau_{i, m}\right) c_{j}^{*}\left(t-\Delta_{i}\right) d t=\rho_{m, j}\left(\frac{\Delta_{i}-\tau_{i, m}}{T_{s}}\right), & \tau_{i, m}<\Delta_{i} \\
0, & \text { otherwise }
\end{array},\right.
\end{aligned}
$$

respectively, where $\boldsymbol{R}_{i}(l)=0, \forall|l|>1, \boldsymbol{R}_{i}(l)=\boldsymbol{R}_{i}^{T}(-l)$, and as before, it is assumed that $\rho_{m, j}=\rho$ for $m \neq i$. Furthermore, the time shifts are assumed to be uniformly distributed as $\left(\tau_{i, m}-\Delta_{i}\right) \sim U\left[-\Delta T_{s} / 2, \Delta T_{s} / 2\right]$ around the reference clock $\Delta_{i}, \forall m \in\{1,2, \ldots, N\}_{m \neq i}$, where $\Delta T_{s} \in\left[0, T_{s}\right)$ is the maximum time-shift value. Intuitively, the smaller are the time shifts, the less severe are the timing synchronization errors. Now, let $\boldsymbol{R}_{i}(0)=\overline{\boldsymbol{R}}_{i}$ be defined as

$\overline{\boldsymbol{R}}_{i}=\left[\begin{array}{cccccc}1 & \cdots & \bar{\rho}_{1,(i-1)}^{(0)} & \bar{\rho}_{1,(i+1)}^{(0)} & \cdots & \bar{\rho}_{1, N}^{(0)} \\ \vdots & \ddots & \vdots & \vdots & \cdots & \vdots \\ \bar{\rho}_{(i-1), 1}^{(0)} & \cdots & 1 & \bar{\rho}_{(i-1),(i+1)}^{(0)} & \cdots & \bar{\rho}_{(i-1), N}^{(0)} \\ \bar{\rho}_{(i+1), 1}^{(0)} & \cdots & \bar{\rho}_{(i+1),(i-1)}^{(0)} & 1 & \cdots & \bar{\rho}_{(i+1), N}^{(0)} \\ \vdots & \cdots & \vdots & \vdots & \ddots & \vdots \\ \bar{\rho}_{N, 1}^{(0)} & \cdots & \bar{\rho}_{N,(i-1)}^{(0)} & \bar{\rho}_{N,(i+1)}^{(0)} & \cdots & 1\end{array}\right]$ 
and $\boldsymbol{R}_{i}(1)=\boldsymbol{R}_{i}^{T}(-1)=\tilde{\boldsymbol{R}}_{i}$, where $\tilde{\boldsymbol{R}}_{i}$ is defined as follows [29]

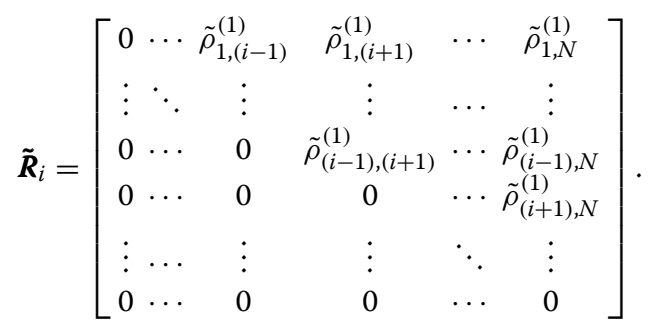

Thus, the output of the matched filter bank can be expressed as $[27,29]$

$$
\mathcal{Y}_{i}(l)=\tilde{\boldsymbol{R}}_{i}^{T} \boldsymbol{A}_{i} \boldsymbol{x}_{i}(l+1)+\overline{\boldsymbol{R}}_{i} \boldsymbol{A}_{i} \boldsymbol{x}_{i}(l)+\tilde{\boldsymbol{R}}_{i} \boldsymbol{A}_{i} \boldsymbol{x}_{i}(l-1)+\overline{\boldsymbol{w}}_{i}(l),
$$

where matrix $\boldsymbol{A}_{i}$ is defined in (13), whereas $\boldsymbol{x}_{i}(l+\varsigma)$ is defined in general as

$$
\begin{aligned}
\boldsymbol{x}_{i}(l+\varsigma)= & {\left[x_{1}(l+\varsigma), \ldots, x_{i-1}(l+\varsigma),\right.} \\
& \left.x_{i+1}(l+\varsigma), \ldots, x_{N}(l+\varsigma)\right]^{T}
\end{aligned}
$$

for $\varsigma \in\{-1,0,1\}$. Also, $\bar{w}_{i}(l)$ is the noise vector with variance given by

$$
\begin{aligned}
E & {\left[\overline{\boldsymbol{w}}_{i}(l) \overline{\boldsymbol{w}}_{i}^{H}(l+\varsigma)\right] } \\
& =\left\{\begin{array}{ll}
N_{0}\left(\left(\mathbf{I}+\boldsymbol{G}_{i}\right) \overline{\boldsymbol{R}}_{i}^{T}+\boldsymbol{G}_{i} \tilde{\boldsymbol{R}}_{i}\right), & \text { if } \varsigma=1 \\
N_{0}\left(\left(\mathbf{I}+\boldsymbol{G}_{i}\right) \overline{\boldsymbol{R}}_{i}+\boldsymbol{G}_{i} \tilde{\boldsymbol{R}}_{i}^{T}+\boldsymbol{G}_{i} \tilde{\boldsymbol{R}}_{i}\right), & \text { if } \varsigma=0 \\
N_{0}\left(\left(\mathbf{I}+\boldsymbol{G}_{i}\right) \overline{\boldsymbol{R}}_{i}+\boldsymbol{G}_{i} \tilde{\boldsymbol{R}}_{i}^{T}\right), & \text { if } \varsigma=-1 \\
0, & \text { otherwise }
\end{array},\right.
\end{aligned}
$$

where $E[\cdot]$ is the expectation operator, and matrix $G_{i}$ is defined in (14). As before, the vector $\mathcal{Y}_{i}(l)$ can be decorrelated as $\overline{\mathcal{Y}}_{i}(l)=\boldsymbol{R}_{i}^{-1} \mathcal{Y}_{i}(l)=\hat{\boldsymbol{R}}_{i} \boldsymbol{A}_{i} \boldsymbol{x}_{i}(l+1)+$ $\overline{\overline{\boldsymbol{R}}}_{i} \boldsymbol{A}_{i} \boldsymbol{x}_{i}(l)+\grave{\boldsymbol{R}}_{i} \boldsymbol{A}_{i} \boldsymbol{x}_{i}(l-1)+\overline{\overline{\boldsymbol{w}}}_{i}(l)$, where $\boldsymbol{R}_{i}$ is as defined in (12) with off-diagonal elements equal to $\rho, \overline{\overline{\boldsymbol{R}}}_{i}=\boldsymbol{R}_{i}^{-1} \overline{\boldsymbol{R}}_{i}$, $\tilde{\boldsymbol{R}}_{i}=\boldsymbol{R}_{i}^{-1} \tilde{\boldsymbol{R}}_{i}^{T}, \grave{\boldsymbol{R}}_{i}=\boldsymbol{R}_{i}^{-1} \tilde{\boldsymbol{R}}_{i}$, and $\overline{\overline{\boldsymbol{w}}}_{i}(l)=\boldsymbol{R}_{i}^{-1} \overline{\boldsymbol{w}}_{i}(l)$ with

$$
\begin{aligned}
E & {\left[\overline{\overline{\boldsymbol{w}}}_{i}(l) \overline{\overline{\boldsymbol{w}}}_{i}^{H}(l+\varsigma)\right] } \\
& =\left\{\begin{array}{ll}
N_{0} \boldsymbol{R}_{i}^{-1}\left(\left(\mathbf{I}+\boldsymbol{G}_{i}\right) \overline{\boldsymbol{R}}_{i}^{T}+\boldsymbol{G}_{i} \tilde{\boldsymbol{R}}_{i}\right) \boldsymbol{R}_{i}^{-T}, & \text { if } \varsigma=1 \\
N_{0} \boldsymbol{R}_{i}^{-1}\left(\left(\mathbf{I}+\boldsymbol{G}_{i}\right) \overline{\boldsymbol{R}}_{i}+\boldsymbol{G}_{i} \tilde{\boldsymbol{R}}_{i}^{T}+\boldsymbol{G}_{i} \tilde{\boldsymbol{R}}_{i}\right) \boldsymbol{R}_{i}^{-T}, & \text { if } \varsigma=0 \\
N_{0} \boldsymbol{R}_{i}^{-1}\left(\left(\mathbf{I}+\boldsymbol{G}_{i}\right) \overline{\boldsymbol{R}}_{i}+\boldsymbol{G}_{i} \tilde{\boldsymbol{R}}_{i}^{T}\right) \boldsymbol{R}_{i}^{-T}, & \text { if } \varsigma=-1 \\
0, & \text { otherwise }
\end{array} .\right.
\end{aligned}
$$

The decorrelated received signal $\overline{\mathcal{Y}}_{j, i}(l)$ at the output of the $j$ th MFB branch is given by where $\overline{\bar{\rho}}_{j, j}$, is the $j$ th diagonal element of matrix $\overline{\overline{\boldsymbol{R}}}_{i}$, while $\dot{\rho}_{j, m}$ and $\grave{\rho}_{j, m}$ are the $(j, m)$ th element of matrices $\hat{\boldsymbol{R}}_{i}$ and $\grave{\boldsymbol{R}}_{i}$, respectively. Additionally, $\overline{\bar{w}}_{j, i}(l) \sim$ $\mathcal{C N}\left(0, \overline{\bar{\varrho}}_{j, i} N_{0}\right)$, where $\overline{\bar{\varrho}}_{j, i}$ is the $j$ th diagonal element of matrix $\boldsymbol{R}_{i}^{-1}\left(\left(\mathbf{I}+\boldsymbol{G}_{i}\right) \overline{\boldsymbol{R}}_{i}+\boldsymbol{G}_{i} \tilde{\boldsymbol{R}}_{i}^{T}+\boldsymbol{G}_{i} \tilde{\boldsymbol{R}}_{i}\right) \boldsymbol{R}_{i}^{-T}$. Based on the above analysis, the instantaneous conditional signalto-interference-plus-noise ratio (SINR) at the output of the MRC of node $S_{i}$ of symbol $x_{j}$ for $j \neq i$, after further manipulation, is obtained as

$$
\begin{aligned}
\gamma_{j, i}= & \gamma_{j, i}^{\mathrm{BP}}+\hat{\gamma}_{j, i}^{\mathrm{CP}}=\frac{P_{j}^{\mathrm{B}}\left|h_{j, i}\right|^{2}}{N_{0}} \\
& +\frac{P_{j}^{\mathrm{B}}\left(\sum_{\substack{k=1 \\
k \neq i, k \neq j}}^{N} \frac{P_{j, k, i}^{\mathrm{C}}\left|h_{j, k}\right|^{2}\left|h_{k, i}\right|^{2}}{P_{j}^{\mathrm{B}}\left|h_{j, k}\right|^{2}+N_{0}}\right) \overline{\bar{\rho}}_{j, j}^{2}}{\mathcal{I}_{j, i}+\overline{\bar{\varrho}}_{j, i} N_{0}},
\end{aligned}
$$

where $\mathcal{I}_{j, i}$ is the ISI variance as defined by

$$
\begin{aligned}
\mathcal{I}_{j, i}= & \sum_{m=1, m \neq i}^{N} P_{m}^{\mathrm{B}}\left(\sum_{\substack{k=1 \\
k \neq i, k \neq m}}^{N} \frac{P_{m, k, i}^{\mathrm{C}}\left|h_{m, k}\right|^{2}\left|h_{k, i}\right|^{2}}{P_{m}^{\mathrm{B}}\left|h_{m, k}\right|^{2}+N_{0}}\right)\left(\dot{\rho}_{j, m}^{2}+\grave{\rho}_{j, m}^{2}\right) \\
& +\sum_{m=1, m \neq i, j}^{N} P_{m}^{\mathrm{B}}\left(\sum_{\substack{k=1 \\
k \neq i, k \neq m}}^{N} \frac{P_{m, k, i}^{\mathrm{C}}\left|h_{m, k}\right|^{2}\left|h_{k, i}\right|^{2}}{P_{m}^{\mathrm{B}}\left|h_{m, k}\right|^{2}+N_{0}}\right) \overline{\bar{\rho}}_{j, m}^{2},
\end{aligned}
$$

and it is assumed that the data symbols are statistically independent. Based on (62), finding a closed form solution for the SER for M-PSK modulation is extremely difficult; therefore, a conditional SER given the channel knowledge is obtained by substituting (62) into (34) and then numerically evaluating it.

It should be noted that $\gamma_{j, i}$ in (62) is composed of the SNR due to the broadcasting phase and the SINR due to the cooperation phase. Thus, it can be verified that if $\tau_{i, m}-\Delta_{i}=0, \forall i, m \in\{1,2, \ldots, N\}$ and $i \neq m$ (i.e., perfect timing synchronization), then $\bar{\rho}_{j, i}^{(0)}=\rho_{j, i}$ and also $\tilde{\rho}_{j, i}^{(-1)}=\tilde{\rho}_{j, i}^{(1)}=0$ and thus the SINR $\gamma_{j, i}$ in (62) reduces to that of (17), as $\hat{\gamma}_{j, i}^{\mathrm{CP}}$ in (62) reduces to the one in (18).

$$
\overline{\mathcal{Y}}_{j, i}(l)=\underbrace{\alpha_{j, i} \overline{\bar{\rho}}_{j, j} x_{j}(l)}_{\text {Desired symbol }}+\underbrace{\sum_{m=1, m \neq i}^{N} \alpha_{m, i}\left(\dot{\rho}_{j, m} x_{m}(l+1)+\dot{\rho}_{j, m} x_{m}(l-1)\right)+\sum_{m=1, m \neq i, j}^{N} \alpha_{m, i} \overline{\bar{\rho}}_{j, m} x_{m}(l)}_{\text {ISI symbols }}+\overline{\bar{w}}_{j, i}(l),
$$




\subsection{Signal model under M2M-STNC-ONS scheme}

From (23), the received signal at node $S_{i}$ is given by

$$
\hat{\mathcal{Y}}_{i}(t)=\sum_{m=1, m \neq i}^{N} \hat{\alpha}_{m, i} \sum_{l=-1}^{1} x_{m}(l) c_{m}\left(t-l T_{s}-\tau_{i, m}\right)+\bar{w}_{i}(t),
$$

where $\hat{\alpha}_{m, i}$ is defined in (24) and $\hat{w}_{i}(t)$ is written as

$$
\begin{aligned}
\hat{w}_{i}(t)= & w_{i}(t)+\sum_{m=1, m \neq i}^{N} \beta_{m, \mathrm{opt}, i} \hat{h}_{m, \mathrm{opt}, i} \\
& \times \sum_{l=-1}^{1} n_{m, \mathrm{opt}, i}\left(t-l T_{s}\right) c_{m}\left(t-l T_{s}-\tau_{i, m}\right) .
\end{aligned}
$$

Following the analysis of the M2M-STNC scheme and replacing matrices $\boldsymbol{A}_{i}$ and $\boldsymbol{G}_{i}$ with $\hat{\boldsymbol{A}}_{i}$ and $\hat{\boldsymbol{G}}_{i}$, respectively (see (26) and (27)), the instantaneous conditional SINR of symbol $x_{j}$ at node $S_{i}$ can be shown to be

$$
\hat{\gamma}_{j, i}=\frac{P_{j}^{\mathrm{B}}\left|h_{j, i}\right|^{2}}{N_{0}}+\frac{P_{j}^{\mathrm{B}}\left(\frac{P_{j, i}^{\mathrm{C}}\left|\hat{h}_{j, \mathrm{opt}, i}\right|^{2}\left|\bar{h}_{j, \mathrm{opt}, i}\right|^{2}}{P_{j}^{\mathrm{B}}\left|\bar{h}_{j, \mathrm{opt}, i}\right|^{2}+N_{0}}\right) \overline{\bar{\rho}}_{j, j}^{2}}{\hat{\mathcal{I}}_{j, i}+\hat{\hat{\varrho}}_{j, i} N_{0}},
$$

where $\hat{\hat{Q}}_{j, i}$ is the $j$ th diagonal element of matrix $\boldsymbol{R}_{i}^{-1}\left(\left(\mathbf{I}+\hat{\boldsymbol{G}}_{i}\right) \overline{\boldsymbol{R}}_{i}+\hat{\boldsymbol{G}}_{i} \tilde{\boldsymbol{R}}_{i}^{T}+\hat{\boldsymbol{G}}_{i} \tilde{\boldsymbol{R}}_{i}\right) \boldsymbol{R}_{i}^{-T}$, and

$$
\begin{aligned}
\hat{\mathcal{I}}_{j, i}= & \sum_{m=1, m \neq i}^{N} P_{m}^{\mathrm{B}}\left(\frac{P_{m, i}^{\mathrm{C}}\left|\hat{h}_{m, \mathrm{opt}, i}\right|^{2}\left|\bar{h}_{m, \mathrm{opt}, i}\right|^{2}}{P_{m}^{\mathrm{B}}\left|\bar{h}_{m, \mathrm{opt}, i}\right|^{2}+N_{0}}\right)\left(\dot{\rho}_{j, m}^{2}+\grave{\rho}_{j, m}^{2}\right) \\
& +\sum_{m=1, m \neq i, j}^{N} P_{m}^{\mathrm{B}}\left(\frac{P_{m, i}^{\mathrm{C}}\left|\hat{h}_{m, \mathrm{opt}, i}\right|^{2}\left|\bar{h}_{m, \mathrm{opt}, i}\right|^{2}}{P_{m}^{\mathrm{B}}\left|\bar{h}_{m, \mathrm{opt}, i}\right|^{2}+N_{0}}\right) \overline{\bar{\rho}}_{j, m}^{2} \cdot s
\end{aligned}
$$

It is noteworthy that under perfect timing synchronization, (66) reduces to (29), as the SINR term due to the cooperation phase reduces to the SNR term of (30).

\section{Imperfect channel state information}

So far, perfect CSI has been assumed and in practice, such assumption is not valid. Channel estimation errors are possibly caused by inaccurate channel estimation/equalization, noise or Doppler shift. Conventionally, channel estimation is based on transmitting a known pilot 'training' sequence with a particular power, prior to data transmission. Inaccurate channel estimation results in a channel estimation error with variance, denoted as $\varepsilon$. At the end of the training phase, the receiving node has imperfect CSI for channel equalization and data detection. In the following subsections, the impact of channel estimation errors on the performance of the M2M-STNC and M2M-STNC-ONS schemes, assuming perfect timing synchronization, is studied and characterized.

\subsection{M2M-STNC}

In the broadcasting phase, the received signal at node $S_{i}$ from node $S_{j}$ with channel estimation error is expressed as

$$
y_{j, i}^{\varepsilon}(t)=\sqrt{P_{j}^{\mathrm{B}}}\left(h_{j, i}+h_{j, i}^{\varepsilon}\right) x_{j} c_{j}(t)+n_{j, i}(t)
$$

where $h_{j, i}^{\varepsilon}$ denotes the channel estimation error. Consequently, $\sqrt{P_{j}^{\mathrm{B}}} h_{j, i}^{\varepsilon} x_{j}$ is the added noise term that scales with the broadcasting power. Furthermore, the channel estimation error $h_{j, i}^{\varepsilon}$ is modeled as a zero-mean complex Gaussian random variable with variance $\varepsilon_{j, i}$. Thus, the additional self-noise term $\sqrt{P_{j}^{\mathrm{B}}} h_{j, i}^{\varepsilon} x_{j}$ is a zero-mean complex Gaussian random variable with variance $\varepsilon_{j, i} P_{j}^{\mathrm{B}}$. Equation (68) is re-written as

$$
y_{j, i}^{\varepsilon}(t)=\sqrt{P_{j}^{\mathrm{B}}} h_{j, i} x_{j} c_{j}(t)+n_{j, i}^{\varepsilon}(t)
$$

where $n_{j, i}^{\varepsilon}(t)=\sqrt{P_{j}^{\mathrm{B}}} h_{j, i}^{\varepsilon} x_{j} c_{j}(t)+n_{j, i}(t)$ is a zero-mean Gaussian random variable with variance $\varepsilon_{j, i} P_{j}^{\mathrm{B}}+N_{0}$. Thus, the SNR after matched filtering is given by

$$
\gamma_{j, i}^{\mathrm{BP}}=\frac{P_{j}^{\mathrm{B}}\left|h_{j, i}\right|^{2}}{\varepsilon_{j, i} P_{j}^{\mathrm{B}}+N_{0}} .
$$

In the cooperation phase, the received signal at node $S_{i}$ is given by

$$
\mathcal{Y}_{i}^{\varepsilon}(t)=\sum_{m=1, m \neq i}^{N} \alpha_{m, i}^{\varepsilon} x_{m} c_{m}(t)+\bar{w}_{i}^{\varepsilon}(t)
$$

where $\alpha_{m, i}^{\varepsilon}$ is defined as

$$
\alpha_{m, i}^{\varepsilon}=\sqrt{P_{m}^{\mathrm{B}}} \sum_{\substack{k=1 \\ k \neq i, k \neq m}}^{N} \beta_{m, k, i}^{\varepsilon} h_{m, k} h_{k, i}
$$

and

$$
\beta_{m, k, i}^{\varepsilon}=\sqrt{\frac{P_{m, k, i}^{\mathrm{C}}}{P_{m}^{\mathrm{B}}\left(\left|h_{m, k}\right|^{2}+\varepsilon_{m, k}\right)+N_{0}}} .
$$

As before, $w_{i}(t)$ is the zero-mean $N_{0}$-variance AWGN sample at node $S_{i}$ and $\bar{w}_{i}^{\varepsilon}(t)$ is the equivalent noise term, expressed as 


$$
\bar{w}_{i}^{\varepsilon}(t)=w_{i}(t)+\sum_{m=1, m \neq i}^{N} \sum_{\substack{k=1 \\ k \neq i, k \neq m}}^{N} \beta_{m, k, i}^{\varepsilon}\left(\left(h_{k, i}+h_{k, i}^{\varepsilon}\right) n_{m, k}+\left(h_{k, i} h_{m, k}^{\varepsilon}+h_{m, k} h_{k, i}^{\varepsilon}+h_{m, k}^{\varepsilon} h_{k, i}^{\varepsilon}\right) x_{m}\right) c_{m}(t) .
$$

After multinode signal detection, the received signal corresponding to symbol $x_{j}$ is given by

$$
\overline{\mathcal{Y}}_{j, i}^{\varepsilon}=\sqrt{P_{j}^{\mathrm{B}}}\left(\sum_{\substack{k=1 \\ k \neq i, k \neq j}}^{N} \beta_{j, k, i}^{\varepsilon} h_{j, k} h_{k, i}\right) x_{j}+\overline{\bar{w}}_{j, i}^{\varepsilon}
$$

where $\overline{\bar{w}}_{j, i}^{\varepsilon} \sim \mathcal{C N}\left(0, \varrho_{N-1} \bar{g}_{j, i}^{\varepsilon}\right)$ and

$$
\bar{g}_{j, i}^{\varepsilon}=N_{0}\left(1+\sum_{\substack{k=1 \\ k \neq i, k \neq j}}^{N}\left(\beta_{j, k, i}^{\varepsilon}\right)^{2}\left(\left|h_{k, i}\right|^{2}+\varepsilon_{k, i}\right)\right)+\sum_{\substack{k=1 \\ k \neq i, k \neq j}}^{N}\left(\beta_{j, k, i}^{\varepsilon}\right)^{2}\left(\left|h_{k, i}\right|^{2} \varepsilon_{j, k}+\left|h_{j, k}\right|^{2} \varepsilon_{k, i}+\varepsilon_{j, k} \varepsilon_{k, i}\right) .
$$

In turn, the SNR at the output of the matched filter of node $S_{i}$ is expressed as

$$
\gamma_{j, i}^{\mathrm{CP}}=\frac{P_{j}^{\mathrm{B}}\left(\sum_{\substack{k=1 \\ k \neq i, k \neq j}}^{N} \frac{P_{j, k, i}^{\mathrm{C}}\left|h_{j, k}\right|^{2}\left|h_{k, i}\right|^{2}}{P_{j}^{\mathrm{B}}\left(\left|h_{j, k}\right|^{2}+\varepsilon_{j, k}\right)+N_{0}}\right)}{\varrho_{N-1}\left(N_{0}\left(1+\sum_{\substack{k=1 \\ k \neq i, k \neq j}}^{N} \frac{P_{j, k, i}^{\mathrm{C}}\left(\left|h_{k, i}\right|^{2}+\varepsilon_{k, i}\right)}{P_{j}^{\mathrm{B}}\left(\left|h_{j, k}\right|^{2}+\varepsilon_{j, k}\right)+N_{0}}\right)+\sum_{\substack{k=1 \\ k \neq i, k \neq j}}^{N} \frac{P_{j, k, i}^{\mathrm{C}}\left(\left|h_{k, i}\right|^{2} \varepsilon_{j, k}+\left|h_{j, k}\right|^{2} \varepsilon_{k, i}+\varepsilon_{j, k} \varepsilon_{k, i}\right)}{P_{j}^{\mathrm{B}}\left(\left|h_{j, k}\right|^{2}+\varepsilon_{j, k}\right)+N_{0}}\right)} .
$$

Based on (70) and (77), it is clear that channel estimation errors increase the noise variance, which in turn reduces the resulting SNR at the output of the matched filter. Thus, with channel estimation errors, increasing the broadcasting and/or transmit power cannot indefinitely increase the SNR. Additionally, in the case of perfect CSI (i.e., $\varepsilon_{j, i}=0$, $\forall j, i \in\{1,2, \ldots, N\}$, and $j \neq i$ ), then the SNR expressions in (70) and (77) reduce to (4) and (18), respectively.

\subsection{M2M-STNC-ONS}

Under the M2M-STNC-ONS scheme ${ }^{\mathrm{d}}$, the received signal (see (23)) in the cooperation phase at node $S_{i}$ is given by

$$
\hat{\mathcal{Y}}_{i}(t)=\sum_{m=1, m \neq i}^{N} \hat{\alpha}_{m, i}^{\varepsilon} x_{m} c_{m}(t)+\hat{w}_{i}^{\varepsilon}(t)
$$

where $\hat{\alpha}_{m, i}^{\varepsilon}$ is defined as

$$
\hat{\alpha}_{m, i}^{\varepsilon}=\beta_{m, \mathrm{opt}, i}^{\varepsilon} \bar{h}_{m, \mathrm{opt}, i} \sqrt{P_{m}^{\mathrm{B}}} \hat{h}_{m, \mathrm{opt}, i} .
$$

Moreover, $\hat{w}_{i}^{\varepsilon}(t)$ is equivalent interference plus noise term, defined as

$$
\hat{w}_{i}^{\varepsilon}(t)=w_{i}(t)+\sum_{m=1, m \neq i}^{N} \beta_{m, \mathrm{opt}, i}^{\varepsilon}\left(\left(\hat{h}_{m, \mathrm{opt}, i}+\hat{h}_{m, \mathrm{opt}, i}^{\varepsilon}\right) n_{m, \mathrm{opt}, i}+\left(\hat{h}_{m, \mathrm{opt}, i} \bar{h}_{m, \mathrm{opt}, i}^{\varepsilon}+\bar{h}_{m, \mathrm{opt},} \hat{h}_{m, \mathrm{opt}, i}^{\varepsilon}+\hat{h}_{m, \mathrm{opt}, i}^{\varepsilon} \bar{h}_{m, \mathrm{opt}, i}^{\varepsilon}\right) x_{m}\right) c_{m}(t) .
$$

Following (28), the decorrelated signal can be obtained as

$$
\hat{\mathcal{Y}}_{j, i}^{\varepsilon}=\beta_{j, \mathrm{opt}, i}^{\varepsilon} \bar{h}_{j, \mathrm{opt}, i} \sqrt{P_{j}^{\mathrm{B}}} \hat{h}_{j, \mathrm{opt}, i} x_{j}+\hat{\hat{w}}_{j, i}^{\varepsilon},
$$

where $\hat{\hat{w}}_{j, i}^{\varepsilon} \sim \mathcal{C N}\left(0, \varrho_{N-1} \hat{g}_{j, i}^{\varepsilon}\right)$, where

$$
\hat{g}_{j, i}^{\varepsilon}=N_{0}\left(1+\left(\beta_{j, \mathrm{opt}, i}^{\varepsilon}\right)^{2}\left(\left|\hat{h}_{j, \mathrm{opt}, i}\right|^{2}+\hat{\varepsilon}_{j, \mathrm{opt}, i}\right)\right)+\left(\beta_{j, \mathrm{opt}, i}^{\varepsilon}\right)^{2}\left(\left|\hat{h}_{j, \mathrm{opt}, i}\right|^{2} \bar{\varepsilon}_{j, \mathrm{opt}, i}+\left|\bar{h}_{j, \mathrm{opt}, i}\right|^{2} \hat{\varepsilon}_{j, \mathrm{opt}, i}+\hat{\varepsilon}_{j, \mathrm{opt}, i} \bar{\varepsilon}_{j, \mathrm{opt}, i}\right) .
$$

Therefore, the SNR at the output of the matched filter is expressed as 


$$
\hat{\gamma}_{j, i}^{\mathrm{CP}}=\frac{\left.P_{j}^{\mathrm{B}} P_{j, i}^{\mathrm{C}} \bar{h}_{j, \mathrm{opt}, i}\right|^{2}\left|\hat{h}_{j, \mathrm{opt}, i}\right|^{2}}{\varrho_{N-1}\left(N_{0}\left(P_{j}^{\mathrm{B}}\left(\left|\bar{h}_{j, \mathrm{opt}, i}\right|^{2}+\bar{\varepsilon}_{j, \mathrm{opt}, i}\right)+P_{j, i}^{\mathrm{C}}\left(\left|\hat{h}_{j, \mathrm{opt}, i}\right|^{2}+\hat{\varepsilon}_{j, \mathrm{opt}, i}\right)+N_{0}\right)+P_{j, i}^{\mathrm{C}}\left(\left|\hat{h}_{j, \mathrm{opt}, i}\right|^{2} \bar{\varepsilon}_{j, \mathrm{opt}, i}+\left|\bar{h}_{j, \mathrm{opt}, i}\right|^{2} \hat{\varepsilon}_{j, \mathrm{opt}, i}+\hat{\varepsilon}_{j, \mathrm{opt}, i} \bar{\varepsilon}_{j, \mathrm{opt}, i}\right)\right)},
$$

where it should be noted that in the perfect CSI case, the SNR expression in (83) reduces to that of (30). Finally, by comparing (77) and (83), it can be seen that the SNR term in (83) is affected only by the channel estimation errors of the channels between node $S_{j}$, the optimally selected node, and the destination node $S_{i}$, rather than the channels between node $S_{j}$ and all the other intermediate nodes as well as node $S_{i}$, for $j \neq i$.

\section{Performance evaluation}

In this section, the analytical derivations of the SER and achievable rate performance of the M2M-STNC and M2M-STNC-ONS schemes are evaluated and compared for node $S_{1}$ 's symbol $x_{1}$ received at node $S_{4}$. Equal power allocation between the two transmission phases is assumed, such that $P_{1}^{\mathrm{B}}=P_{1}^{\mathrm{C}}=P / 2, P_{1, i}^{\mathrm{C}}=P_{1}^{\mathrm{C}} /(N-1)=$ $P /(2(N-1)), \forall i \in\{2, \ldots, N\}$ and $P_{1, k, i}^{C}=P_{1, i}^{C} /(N-2)=$ $P /(2(N-1)(N-2)), \forall k \in\{2, \ldots, N\}$ and $k \neq i$. The network nodes are located as shown in Figure 2 with the channel variance between nodes $S_{j}$ and $S_{i}$ being defined as $h_{j, i} \sim \mathcal{C N}\left(0, d_{j, i}^{-v}\right), \forall j, i \in\{1,2, \ldots, N\}$ for $j \neq i$ and $v=3$. Non-orthogonal signature waveforms with a crosscorrelation of $\rho_{j, i}=\rho=0.5$ for $j \neq i$ are also assumed. The channel estimation error variance is assumed to be the same between any two nodes (i.e., $\varepsilon_{j, i}=\varepsilon, \forall j, i \in$ $\{1,2, \ldots, N\}$ for $j \neq i$.

To verify the achievable diversity order, consider the following cases. When $N=3$, the network consists of nodes $S_{1}, S_{3}$, and $S_{4}$ (i.e., $S_{2}$ and $S_{5}$ are inactive). In

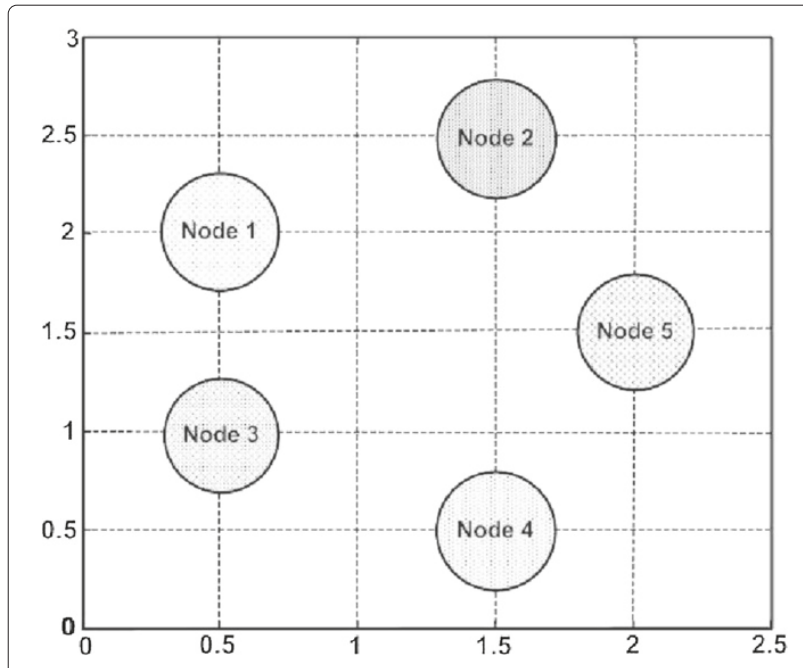

Figure 2 Network topology. this case, only node $S_{3}$ relays symbol $x_{1}$ to node $S_{4}$. As for $N=4$, all network nodes but node $S_{5}$ are active. Hence, nodes $S_{2}$ and $S_{3}$ act as relays for node $S_{1}$. Lastly, for $N=5$, all network nodes are active, in which case nodes $S_{2}, S_{3}$, and $S_{5}$ forward symbol $x_{1}$ to node $S_{4}$. Now, from Figure 3 and assuming perfect timing synchronization and CSI, it is clear that as the number of cooperating nodes $N$ in the network increases, the SER performance of both schemes improves which is due to the increased diversity order achievable with the increase in $N$. Also, for $N=3$, both schemes yield the same SER performance. Moreover, the numerically evaluated SER performance of the M2M-STNC scheme (see Figure 3a) perfectly agrees with its simulated performance. In addition, the SER performance of proposed the M2M-STNC-ONS scheme (see Figure 3b) outperforms that of its counterpart for $N=$ 4 and $N=5$. Furthermore, the derived approximate SER theoretical expression under the M2M-STNC-ONS scheme coincides with the simulated performance except for a slight deviation at low SNR which is attributed to the approximation used in the theoretical analysis that assumed high enough SNR. Also, the derived upper bound happens to be asymptotic at high SNR and thus confirms the achievable diversity order per node.

From this point onwards, only the cases of $N=4$ and $N=5$ are considered. Now, in Figure 4a, the SER performance of the M2M-STNC and M2M-STNCONS schemes is compared with timing synchronization errors and perfect CSI for $N=4$ nodes. It is clear that the M2M-STNC-ONS scheme is more resistant to timing offsets than its counterpart scheme. This is because the number of simultaneous transmissions in each time slot is reduced, and only the node achieving the highest cooperative SNR at the receiving node relays its received signal. A similar observation can be seen in the case of $N=5$ nodes (see Figure 4b). However, it is noticed that the impact of timing offsets on the SER performance is less severe despite the increase in the number of transmitting nodes. This is again due to the increased diversity order.

The performance of the M2M-STNC and M2M-STNCONS schemes with channel estimation errors and perfect timing synchronization for $N=4$ nodes is demonstrated in Figure 5a. It is clear that with the increase in channel estimation error variance $\varepsilon$, the SER performance of both schemes degrades with the M2M-STNC-ONS scheme still significantly outperforming its counterpart scheme. The case of $N=5$ nodes is shown in Figure $5 \mathrm{~b}$ with the SER performance of both schemes being less affected by 

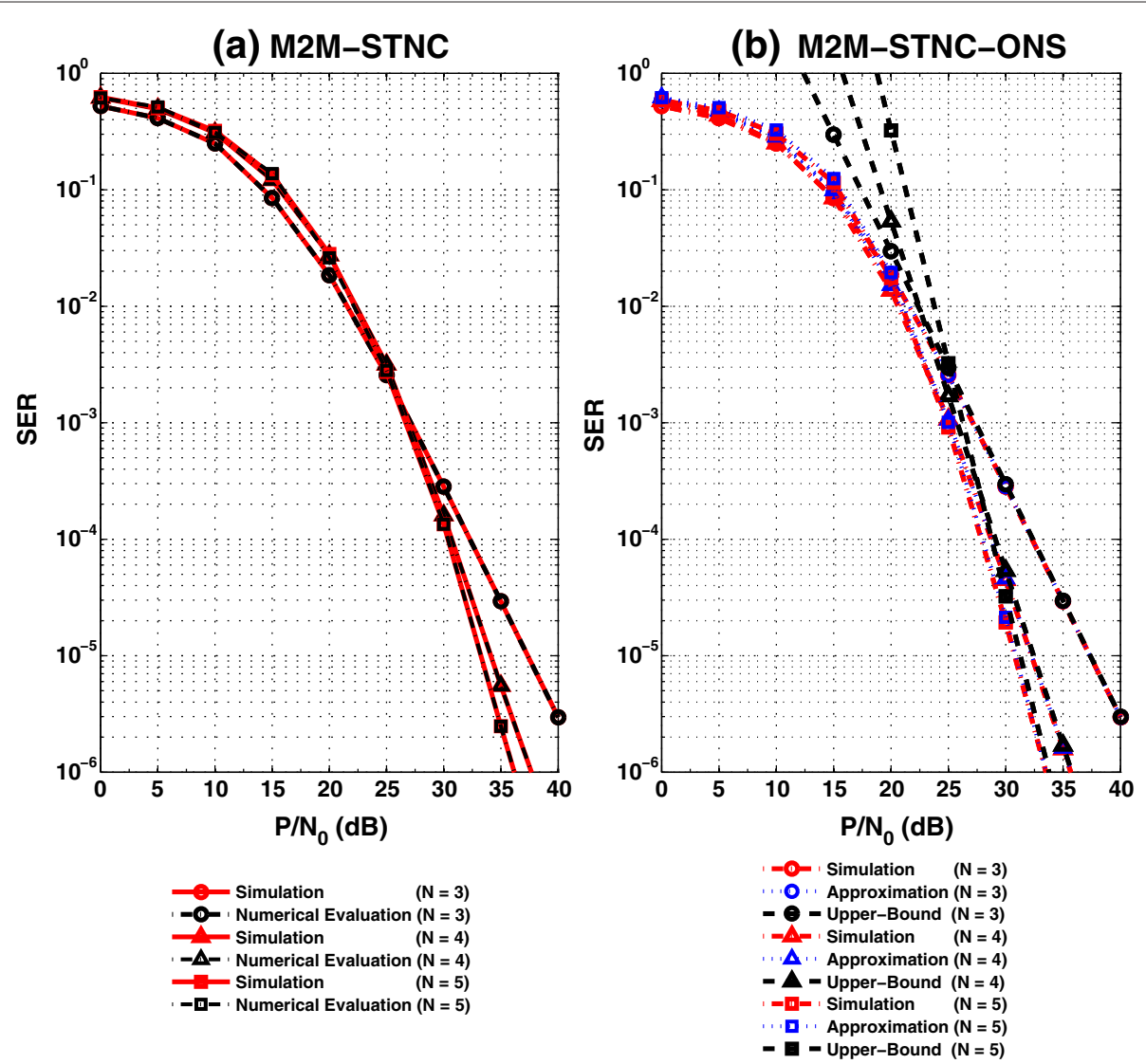

Figure 3 QPSK SER performance of the (a) M2M-STNC and (b) M2M-STNC-ONS schemes - perfect timing synchronization and CSI.

channel estimation errors due to the increased diversity gains, as aforementioned.

In Figure 6a, the achievable rate of node $S_{1}$ at node $S_{4}$ for $N=4$ nodes is illustrated. It is evident that the proposed M2M-STNC-ONS scheme with perfect timing synchronization and CSI achieves a better rate than the M2M-STNC scheme. In general, the achievable rate of node $S_{1}$ at node $S_{4}$ when $N=5$ (see Figure 6b) is less than that when $N=4$, which is due to the increased number of time slots required for communication between all the nodes. The same observation holds for the different cases of perfect/imperfect timing synchronization and CSI.

Figure 7 illustrates the total achievable rate of source node $S_{1}$ for $N=4$ and $N=5$ node networks. It is clear that the proposed M2M-STNC-ONS scheme is superior to the M2M-STNC scheme, and the total achievable rate for a network with $N=5$ is higher than that of a network with $N=4$, despite the decrease in the rate between node $S_{1}$ and all the other nodes in the networks. Finally, the gain in rate of the M2M-STNC-ONS scheme compared with its counterpart M2M-STNC scheme is higher for the network with $N=5$ nodes. This again can be seen from the different cases of perfect/imperfect timing synchronization and CSI.

\section{Conclusions}

In this paper, the problem of multinode cooperative communications has been investigated. In particular, the performance of the M2M-STNC and the M2M-STNC-ONS schemes has been studied and analyzed. It has been shown that both schemes allow $N$ amplify-and-forward nodes to exchange their data symbols simultaneously over a total of $2 N$ time slots. Moreover, an analytical expression for the conditional SER of the M2M-STNC scheme has been provided, while approximate and asymptotic upper-bound SER expressions for the M2M-STNC-ONS scheme have been derived and shown to coincide with the simulated results. The superiority of the M2M-STNCONS scheme with $N$ nodes manifests itself in the fact that only two time slots are effectively required per node to achieve a diversity order of $N-1$ per transmitted symbol while allowing all the $N$ nodes to exchange their data symbols simultaneously over a total of $2 N$ time slots as opposed to the conventional multinode relay networks that require $N^{2}$ time slots to achieve the same diversity order of $N-1$ per node even with optimal relay selection [16]. Also, the M2M-STNC-ONS reduces the total number of transmissions in each time slot in the cooperation phase in comparison with the M2M-STNC scheme, which 

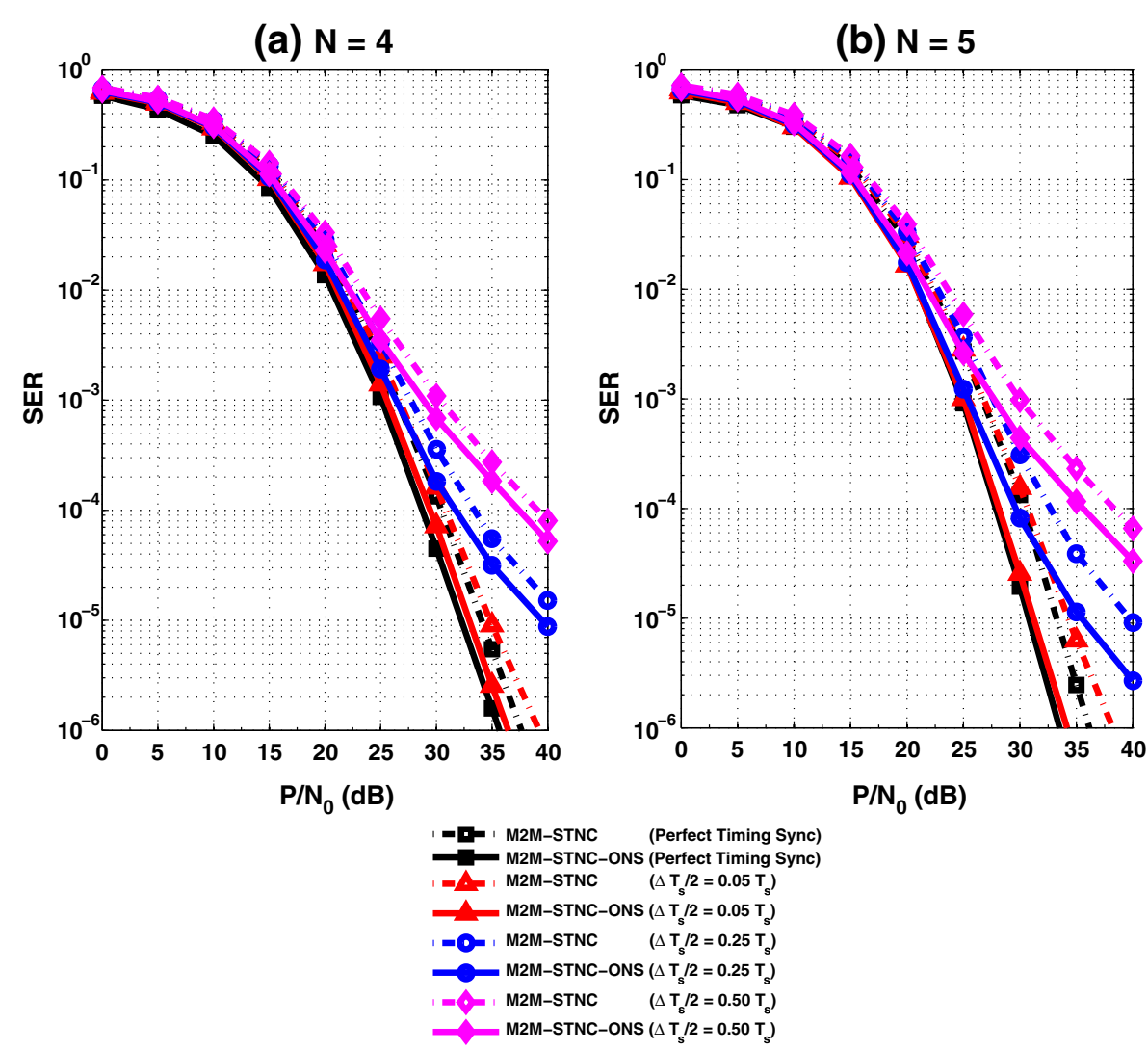

Figure 4 QPSK SER performance with timing synchronization errors for (a) $N=4$ and (b) $N=5$ nodes - perfect CSI.
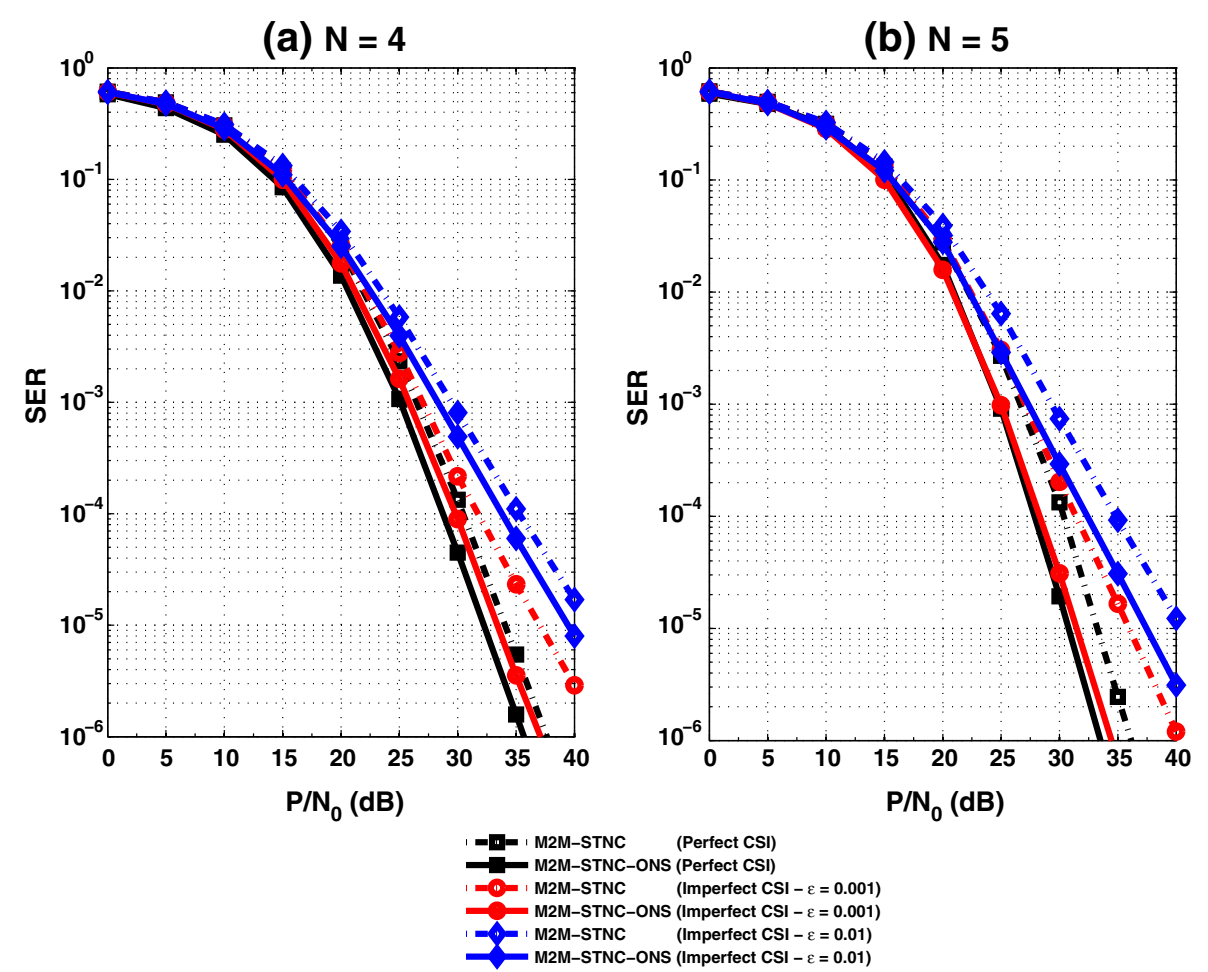

Figure 5 QPSK SER performance with channel estimation errors for (a) $N=4$ and (b) $N=5$ nodes - perfect timing synchronization. 

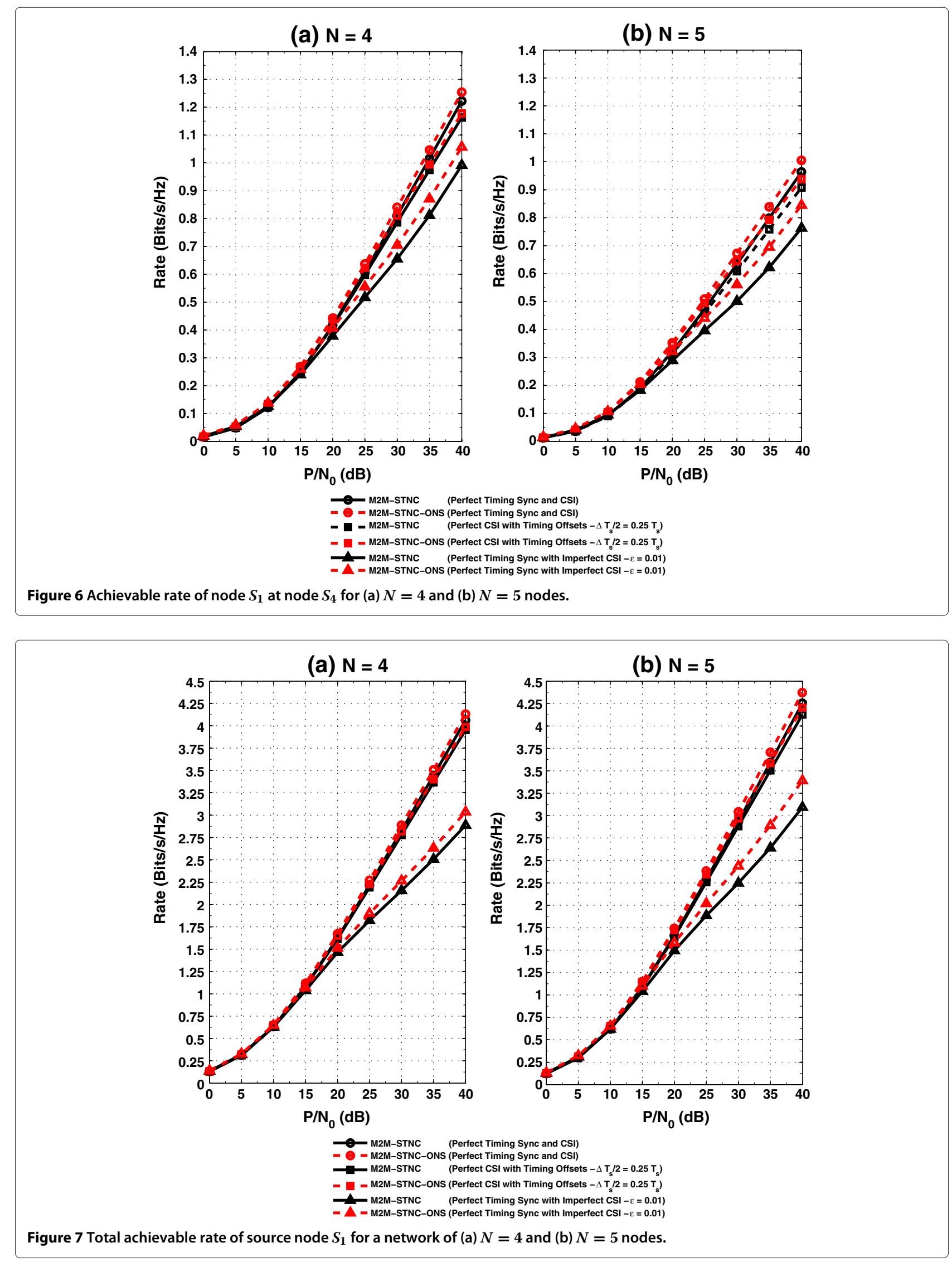
mitigates the effects of noise amplification, timing offsets, and channel estimation errors.

\section{Endnotes}

${ }^{a}$ It should be noted that our many-to-many space-time network coding scheme is a CDMA-like system that operates under conventional TDMA by assigning each network node a signature waveform for single- or multiple-access transmission. Signature waveforms provide immunity against various kinds of noise and multipath distortion, and they are important for multiuser transmission/detection and timing/frequency synchronization at the receiving nodes.

${ }^{\mathrm{b}}$ Node selection is achieved via control messages exchange prior to the cooperation phase [30] and is only updated when the respective channels' coherence time elapses.

${ }^{\mathrm{c}}$ It is assumed that the channel coefficients are time invariant during each time slot but are randomly time varying from one time slot to another.

${ }^{d}$ The node selection criterion is now based on the channel coefficients with estimation errors, which implies that the selected node with imperfect CSI is not necessarily the selected node under perfect CSI.

\section{Competing interests}

The authors declare that they have no competing interests.

\section{Author details}

${ }^{1}$ Electrical Engineering Department, College of Engineering and Petroleum, Kuwait University, Safat 13060, Kuwait. 2Wireless @ Virginia Tech, Bradley Department of Electrical and Computer Engineering, Virginia Tech, Blacksburg, VA 24061, USA.

Received: 8 November 2013 Accepted: 12 March 2014 Published: 26 March 2014

\section{References}

1. R Ahlswede, N Cai, SY Li, RW Yeung, Network information flow. IEEE Trans. Inform. Theory 46, 1204-1216 (2000)

2. KJR Liu, AK Sadek, W Su, A Kwasinski, Cooperative Communications and Networking (Cambridge University Press, Cambridge, 2008)

3. HQ Lai, AS Ibrahim, KJR Liu, Wireless network cocast: location-aware cooperative communications using network coding. IEEE Trans. Wireless Commun. 8, 3844-3854 (2009)

4. TWang, GB Giannakis, Complex field network coding for multiuser cooperative communications. IEEE J. Selected Areas Commun. 26, 561-571 (2008)

5. HQ Lai, KJR Liu, Space-time network coding. IEEE Trans. Signal Process. 4, 1706-1718 (2011)

6. MW Baidas, HQ Lai, KJR Liu, Many-to-many communications via space-time network coding. Paper presented at the IEEE wireless communications and networking conference, Sydney, Australia, 18-21 April 2010, pp. 1-6

7. ZZ Gao, HQ Lai, KJR Liu, Differential space-time network coding for multi-source cooperative communications. IEEE Trans. Commun. 59, 3146-3157 (2011)

8. ZZ Gao, YH Yang, KJR Liu, Anti-eavesdropping space-time network coding for cooperative communications. IEEE Trans. Wireless Commun. 10, 3898-3908 (2011)

9. W Guan, KJR Liu, Clustering based space-time network coding. Paper presented at the IEEE global communications conference (GLOBECOM), Anaheim, CA, USA, 3-7 Dec 2012, pp.5633-5638
10. A Yang, Z Fei, N Yang, C Xing, J Kuang, Symbol error rate of space-time network coding Nakami-m fading. IEEE Trans. Vehicular Technol. 62, 2644-2655 (2013)

11. JN Laneman, GW Wornell, Distributed space-time coded protocols for exploiting cooperative diversity in wireless networks. IEEE Trans. Inform. Theory 49, 2415-2425 (2003)

12. L Venturino, X Wang, M Lops, Multiuser detection for cooperative networks and performance analysis. IEEE Trans. Signal Process. 54, 3315-3329 (2006)

13. S Wei, Diversity-multiplexing tradeoff of asynchronous cooperative diversity in wireless networks. IEEE Trans. Inform. Theory 53, 4150-4172 (2007)

14. AS Ibrahim, KJR Liu, Mitigating channel estimation error with timing synchronization tradeoff in cooperative communications. IEEE Trans. Signal Process. 58, 337-348 (2010)

15. $Y$ Jing, $\mathrm{H}$ Jafarkhani, Single and multiple relay selection schemes and their achievable diversity orders. IEEE Trans. Wireless Commun. 8, 1414-1423 (2009)

16. Y Zhao, R Adve, TJ Lim, Improving amplify-and-forward relay networks: optimal power allocation versus selection. IEEE Trans. Wireless Commun. 6, 3114-3123 (2007)

17. C Sun, W Zhang, KB Letaief, Cluster-based cooperative spectrum sensing in cognitive radio systems. Paper presented at the IEEE international conference on communications (ICC), Glasgow, Scotland, 24-28 June 2007, pp. 2511-2515

18. Z Zhou, S Zhou, S Cui, JH Cui, Energy-efficient cooperative communication in a clustered wireless sensor network. IEEE Trans. Vehicular Technol. 57, 3618-3628 (2008)

19. A Scaglione, D Goeckel, JN Laneman, Cooperative communications in mobile ad-hoc networks: rethinking the link abstraction. IEEE Signal Process. Mag. 23, 18-29 (2006)

20. AS Ibrahim, AK Sadek, W Su, KJR Liu, Cooperative communications with relay-selection: when to cooperate and whom to cooperate with? IEEE Trans. Wireless Commun. 7, 2814-2827 (2008)

21. MO Hasna, MS Alouini, End-to-end performance of transmission systems with relays over rayleigh fading channels. IEEE Trans. Wireless Commun. 2, 1126-1131 (2003)

22. MK Simon, MS Alouini, A unified approach to the performance analysis of digital communications over generalized fading channels. Proc. IEEE 86, 1860-1877 (1998)

23. IS Gradshteyn, IM Ryshik, Table of Integrals, Series and Products, 7th. edn. (Academic, New York, 2007)

24. KG Seddik, AK Sadek, AS Ibrahim, KJR Liu, Design criteria and performance analysis for distributed space-time coding. IEEE Trans. Vehicular Technol. 57, 2280-2292 (2008)

25. X Li, YC Wu, E Serpedin, Timing synchronization in decode-and-forward cooperative communication systems. IEEE Trans. Signal Process. 57, 1444-1455 (2009)

26. S Jagannathan, H Aghajan, A Goldsmith, The effect of time synchronization errors on the performance of cooperative MISO systems. Paper presented at the IEEE global communications (GLOBECOM), Dallas, TX, USA, 29 Nov-3 Dec 2004, pp. 102-107

27. H Elders-Boll, A Bushoom, HD Schotten, Implementation of linear multiuser detectors for asynchronous CDMA systems by linear interference cancellation algorithms. Paper presented at the IEEE vehicular technology conference (VTC), Ottawa, ON, Canada, 18-21 May 1998, pp. 1849-1853

28. KS Kim, I Song, YH Kim, YU Lee, J Lee, Analysis of quasi-ML multiuser detection of DS/CDMA systems in asynchronous channels. IEEE Trans. Commun. 47, 1875-1883 (1999)

29. S Verdu, Multiuser Detection (Cambridge University Press, Cambridge, 1998)

30. H Adam, C Bettstetter, SM Senouci, Adaptive relay selection in cooperative wireless networks. Paper presented at the IEEE international symposium on personal, indoor, and mobile radio communications (PIMRC), Cannes, France, 15-18 Sept 2008, pp. 1-5

doi:10.1186/1687-1499-2014-48

Cite this article as: Baidas and MacKenzie: Many-to-many space-time network coding for amplify-and-forward cooperative networks: node selection and performance analysis. EURASIP Journal on Wireless Communications and Networking 2014 2014:48. 